\title{
New Agile Matrix Antenna for Nano-satellite Telemetry Using Bimodal Pattern Reconfiguration
}

\author{
Ali Siblini ${ }^{1}$, Eric Arnaud ${ }^{2}$, Anthony Bellion ${ }^{3}$, Hussein Abou Taam ${ }^{1}$, Bernard Jecko \\ ${ }^{1}$ Department of Physics and Electronics, Faculty of Sciences, Lebanese University, Beirut, Lebanon \\ ${ }^{2}$ Department RF-Systems, XLIM Research Institute, Limoges University, Limoges, France \\ ${ }^{3}$ Department of Antenna, National Center for Space Studies, Toulouse, France
}

Email address:

ali.siblini.84@hotmail.com (A. Siblini), eric.arnaud@xlim.fr (E. Arnaud), Anthony.Bellion@cnes.fr (A. Bellion), hussein.aboutaam@hotmail.com (H. A. Taam), bernard.jecko@xlim.fr (B. Jecko)

\section{To cite this article:}

Ali Siblini, Eric Arnaud, Anthony Bellion, Hussein Abou Taam, Bernard Jecko. New Agile Matrix Antenna for Nano-satellite Telemetry Using Bimodal Pattern Reconfiguration. American Journal of Science, Engineering and Technology. Special Issue: Machine Learning in the Internet of Things. Vol. 5, No. 1, 2020, pp. 1-19. doi: 10.11648/j.ajset.20200501.11

Received: October 15, 2019; Accepted: November 13, 2019; Published: January 21, 2020

\begin{abstract}
This review deals with the design of a new reconfigurable beam antenna used to improve the efficiency of spatial telemetry links on Nano-Satellite. Advances in this domain show the needs to serve some applications with special electromagnetic beams and polarization patterns especially from LEO and MEO Space-Earth links. The RF front end must be capable to switch from high gain directive pattern to special pattern called Isoflux where the gain must be concentrated at high elevation angles with another challenge also the circular polarization. The content is a part of the CNES project that serves for multimode beam forming applications. This agile beam antenna is not built on the well-known array concept: AESA stands for Agile Electronically Scanned Array but using a new approach called ARMA standing for Agile Radiating Matrix Antenna. Contributions will show the small size elementary antenna of ARMA called Pixel, the polarization circuits that fit the challenges of the Nano-Satellite dimensions, the enhancement in the circular polarization requirements and theoretical and experimental comparison. Special beam forming required in the telemetry applications are achieved with better gain especially for the aim of wide beams. The use of the new approach will show the beam forming advantage of radiating the energy to high elevation angles.
\end{abstract}

Keywords: ARMA (Agile Radiating Matrix Antenna), EBG (Electromagnetic Band-gap Material), FSS (Frequency Selective Surfaces), AESA (Agile Electronically Scanned Arrays), LP (Linear polarization), $\mathrm{CP}$ (Circular Polarization), Isoflux, Bi-mode Pattern

\section{Introduction}

Nano-Satellites are very small platforms whose mass does not exceed $10 \mathrm{Kg}$. They are intended to be used for services which are not desirable to have a large payload. They can also be used to carry out preparatory missions for other more ambitious missions. The goal is for example to validate technologies by reducing risk taking. But the future lies probably in the deployment of 20 to 50 Nano-satellite constellations for distributed measures.

Small satellites are under the research and development in many universities and space agencies for example NASA develops projects of military missions. The major advantage of this platform is that it allows rapid to space: the development times are relatively short and the costs could be considerably lowered to $20 \mathrm{KE} / \mathrm{Kg}$; against $100 \mathrm{kE} / \mathrm{Kg}$; today with the arrival new launcher manufacturers.

Another driving force behind the development of space technologies is the creation of the Nano-satellite standard "Cube-Sat", the first prototypes of which have been deployed in space since 2003. This standard is a modular format consisting of cubes of $10 \mathrm{~cm}$ of edge (format "1U") (Figure 1); Each of these cubes contains its own solar panels, weighs less than $1 \mathrm{Kg}$ and can be assembled in clusters of 2, 3,6 or 12 to compose larger satellites and more powerful, respectively "2U", "3U", "6U" and "12U". These Nanosatellites fly in low orbit, between $200 \mathrm{~km}$ and $700 \mathrm{~km}$ and their short life is about 2 years. 
The speeds offered by UHF/VHF links that were previously used by the platforms Nano-Satellites "Cube-Sat" limit the data rates that can be exchanged with the ground. Power and surfaces available on Nano-Satellites platforms prohibit indeed embarking the strong antennas gain which made it possible to establish high Speed links. To meet the challenge it is envisaged to carry out the X-band link with ground stations a few meters in diameter. To do this, the satellite must have circular polarization antenna, with an Isoflux diagram to optimize the gain during scrolling associated with the low orbit. The development of such an antenna is a key to make the link. This antenna must be integrated on the available area of the platform Cube-Sat $(10 \mathrm{~cm} \times 10 \mathrm{~cm})$. The size of the antenna should use the smallest space possible inside the Cube-Sat volume; mainly surface allowed to the antenna.

These constraints are the keywords of the project proposed by the National Center of Space Study in France (CNES). In the next paragraph the ARMA [1] approach is presented briefly by introducing the theoretical concept, the design of the pixel and the general architecture of the matrix antenna. Then in the rest you will find, the specifications of the project, the solution with the manufacturing steps and simulation-measurements validations.

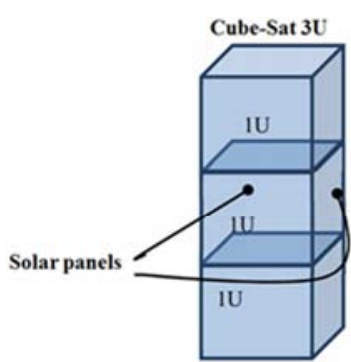

(a)

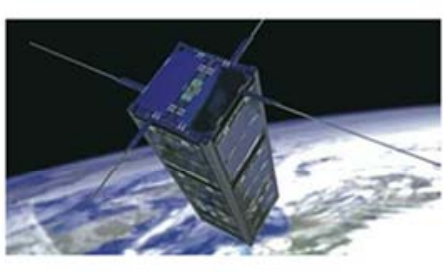

(b)
Figure 1. (a) 3U-type platform, (b) model of a Nano-Satellite with an assembly of 3 cube-Sat with deployable solar panels.

\section{ARMA Electromagnetic Approach}

The radiation pattern of any antenna is obtained by using Maxwell Equations; equations of propagation in free space; free space Green function, and applying the equivalent principle on a radiating surface surrounding the antenna. Figure 2 shows the principle scheme for a planar LP antenna.

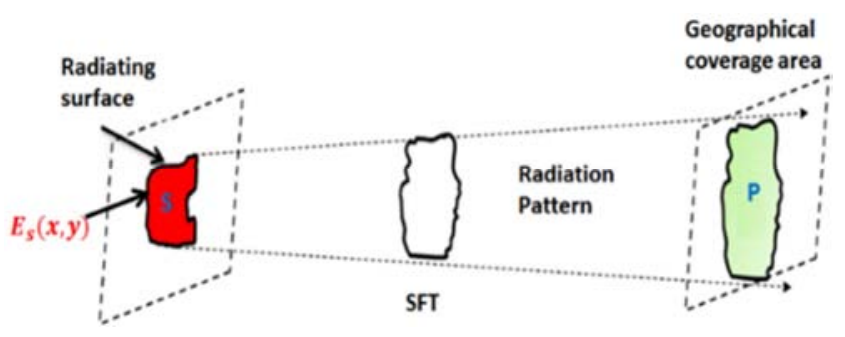

Figure 2. Radiation pattern directly obtained from the radiating surface.

Then the radiated $E M$ field $\vec{E}(P)$ is obtained by a radiating integral like (1):

$$
\begin{gathered}
E(P)=\frac{j k}{4 \pi} \psi(R)(1+\operatorname{Cos} \theta)\left(\operatorname{Sin} \varphi \overrightarrow{e_{\theta}}-\operatorname{Sin} \varphi \overrightarrow{e_{\varphi}}\right) S F T . \\
S F T=\oiint E_{S}(x, y) e^{j\left(k_{x} x \operatorname{Sin} \theta \operatorname{Cos} \varphi+k_{y} y \operatorname{Sin} \theta \operatorname{Sin} \varphi\right)} d s .
\end{gathered}
$$

Where:

$$
\psi(R)=\frac{e^{j k R}}{R} .
$$

This integral relates the radiated electromagnetic field $\vec{E}(P)$ to the field $E_{S}(x, y)$ on the radiating surface $S$, as a quasi-Spatial Fourier Transform (SFT). To obtain a moving radiation pattern the electric surface field must be sampled then this is possible by changing the electric field law $E_{S}(x, y)$ on the radiating surface as shown in Figure 3 .

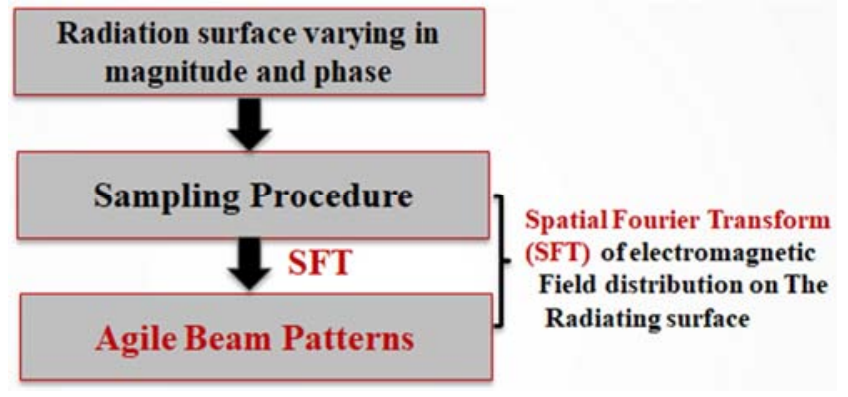

Figure 3. Agile beam patterns related to radiating surface.

\subsection{AESA Sampling Approach}

This well-known procedure "AESA" multiplies the $E_{s}(x, y)$ by a two dimensional Dirac Comb to obtain the sampled form given by the equations below (where $\delta$ is a two dimensional Dirac function). Then the electric radiated field is obtained by the SFT of (4).

$$
\begin{gathered}
\widetilde{E_{S}}(x, y)=\sum_{i} \sum_{j} E_{s}\left(x_{i}, y_{j}\right) \delta_{x_{i}, y_{j}}(x, y) . \\
E(P)=k \iint_{S} \sum_{i} \sum_{j} E_{S}\left(x_{i}, y_{j}\right) \delta_{x_{i}, y_{j}}(x, y) e^{j\left(k_{x} x \operatorname{Sin} \theta \operatorname{Cos} \varphi+k_{y} y \operatorname{Sin} \theta \operatorname{Sin} \varphi\right)} d x d y . \\
E(P)=k \sum_{i} \sum_{j} \iint_{S} E_{S}\left(x_{i}, y_{j}\right) \delta_{x_{i}, y_{j}}(x, y) e^{j\left(k_{x} x \operatorname{Sin} \theta \operatorname{Cos} \varphi+k_{y} y \operatorname{Sin} \theta \operatorname{Sin} \varphi\right)} d x d y .
\end{gathered}
$$

Then the electric radiated field appears as a sum of contributions of punctual elementary antennas periodically distributed on the surface S. That is the Array Approach.

\subsection{Sampling Using Rectangular Function (Quantification)}

The radiating surface $S$ is sampled into small jointed pieces called pixels; the radiated electric field is the sum of all these 
uniform surface field contributions (7), (8) and (9):

$$
\begin{gathered}
E(P)=k \iint_{S} E_{S}(x, y) e^{j(k x \operatorname{Sin} \theta \cos \varphi+k y \operatorname{Sin} \theta \operatorname{Sin} \varphi)} d s . \\
E(P)=k \sum_{i} \sum_{j} \iint_{S i, j} E_{i, j}(x, y) e^{j(k x \operatorname{Sin} \theta \cos \varphi+k y \operatorname{Sin} \theta \operatorname{Sin} \varphi)} d s . \\
E(P)=k \sum_{i} \sum_{j} \iint_{S i, j} A_{i, j} e_{i, j}(x, y) e^{j(k x \operatorname{Sin} \theta \operatorname{Cos} \varphi+k y \operatorname{Sin} \theta \operatorname{Sin} \varphi)} d s=\sum_{i} \sum_{j} A_{i, j} \iint_{S i, j} e_{i, j}(x, y) e^{j(k x \operatorname{Sin} \theta \operatorname{Cos} \varphi+k y \operatorname{Sin} \theta \operatorname{Sin} \varphi)} d s .
\end{gathered}
$$

Where $e_{i, j}$ and $A_{i, j}$ are respectively: The field on the small surfaces considered as a constant field and the weights applied on each normalized pixel surface field $\left(e_{i, j}\right)$.

This formulation suggests that the whole structure can be considered as a matrix of $(M \times N)$ pixels, each able to generate a given contribution to the building of the whole field $\vec{E}(P)$. In the most general cases, there is no restriction on the surface $S$, and the pixels can have any shape, but must be connected together. Like for the arrays, the pixels must be fed by a beam forming network (BFN) to apply appropriate weights in order to obtain an expected radiation pattern. A very important case is to consider Low Profile Antennas. For such structures the surrounding surface is sampled by pixels with a square $s_{i, j}$ radiating surface.

In conclusion, the two approaches introduce different spatial Fourier transform [2] due to the different sampling procedures of the radiating surface. Consequently the performances of these two kinds of antennas ARMA and AESA are different. This is validated in many published works [1-5].

\subsection{Formation of Uniform Radiating Surface}

The most important point in ARMA approach is to be able to design a pixel which can generate a uniform EM field on its surface. This technique, deduced from the knowledge of EBG antennas properties, was extensively developed and validated in many papers $[2,6]$.

The design steps are illustrated in Figure 4, where a high gain EBG antenna (with metallic FSS on the top) shows the circular radiating surface with the maximum at the center and vanishes radially toward the edges. The metallic walls (square shape in this example) are inserted in the zone where the field is uniform; the result is a uniform radiating surface limited by the dimensions of the metallic walls. Then the pixel is a source antenna (patch in this example with), FSS on the top, the metallic walls and the EBG cavity. The patch antenna is used to feed the EBG cavity.
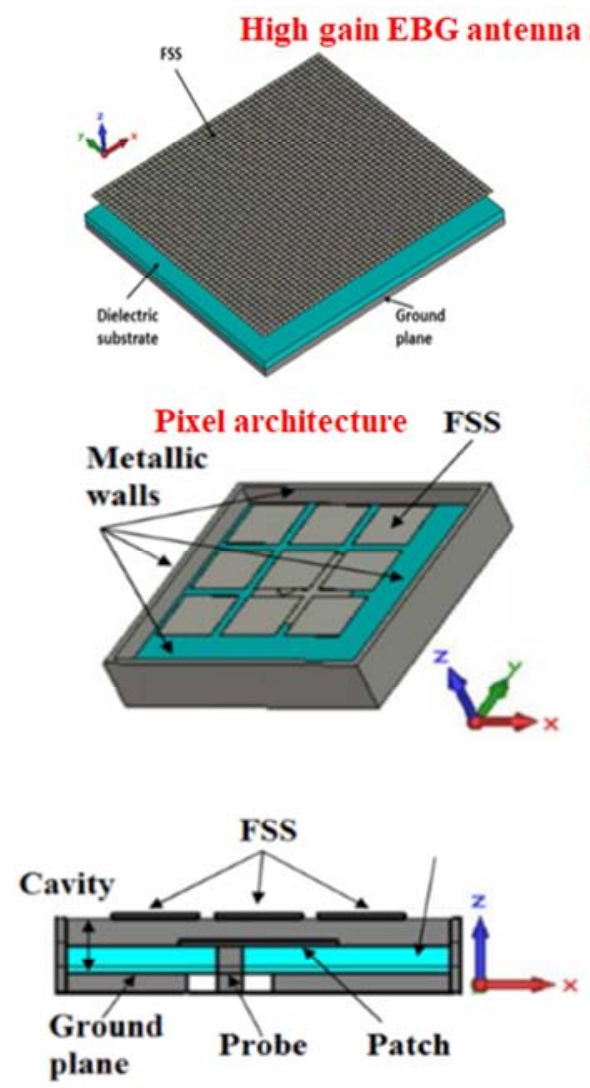

Uniform radiating surface after introducing the metallic walls

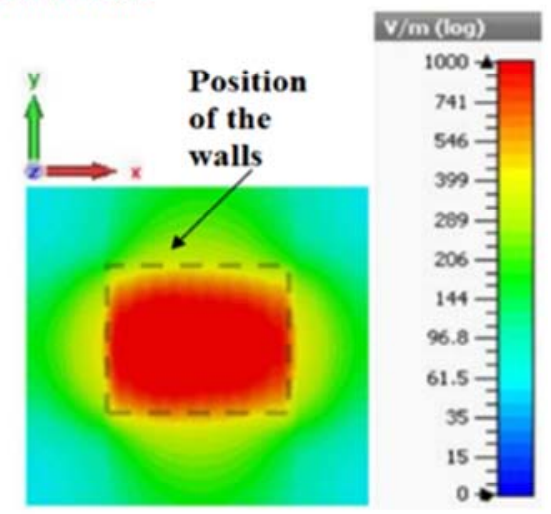

Figure 4. Pixel design: generation of uniform radiating surface.

The corresponding matrix may have many shapes as shown in the Figure 5, 1D planar matrix, 2D planar matrix and conformal matrix. 
1D matrix / M $\times 1$ pixels

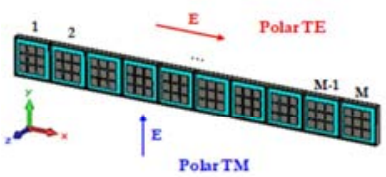

Conformal Matrix / $\mathrm{N} \times \mathrm{M}$ pixels

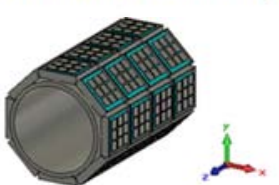

Figure 5. ARMA design: $1 D, 2 D$ and conformal jointed pixels.

\section{CNES Project}

Electronically agile beam antennas are extensively used today to perform beam forming, beam steering, pattern reconfiguration, multi-sectorial beams, tracking etc. For a long time agile beams are obtained using arrays (AESA) of antennas, with a lot of limitations in terms of radiation efficiency, coupling effects, side lobes, back radiation, axial ratio and high steering angles. Recently, a new approach, called ARMA (Agile Radiating Matrix Antenna), was introduced using a periodic quantification function "sampling procedure" to reduce these limitations [7-9].

The CNES call for a project concerns the Nano-satellite. The XLIM (RF-Systems department) have established a partnership respond to the call for project and start worked to resolve the problem using circularly polarized ARMA technology $[9,10]$.

The study consists of designing an agile antenna operating in the $\mathrm{X}$ band [ 8 to 8.4 ] GHz to function with two different beams. First is an Isoflux pattern and second the axial high gain pattern, both with circular polarization for Nano-type satellite platforms Cube-Sat $3 \mathrm{U}$ and $6 \mathrm{U}$. The bandwidth is set at $400 \mathrm{MHz}$ around $8.2 \mathrm{GHz}$. The polarization must be circular on the same band, so axial ratio must be less than 3 $\mathrm{dB}$ in the cone $\theta= \pm 60^{\circ}$. Throughout the study phase, the platforms considered is 3U-type as shown in Figure 6a (This platform is a parallelepiped of $10 \mathrm{~cm} \times 10 \mathrm{~cm} \times 30 \mathrm{~cm}$ ).

The performance of the antenna developed to fulfill the specifications must be impacted as little as possible by the platform and must be optimized with the perturbations generated by the platform. The area reserved for the antenna is a square of $90 \mathrm{~mm}$ of edges and the antenna must not protrude beyond the platform.

The agile antenna is expected to serve two beam modes. The first mode is the wide beam (Isoflux); the Isoflux pattern is used for wide area coverage of the earth from the space where the antenna must radiate power equally for the earth meaning, that the receivers at different distances of the earth should receive the same power. This can explain the fact that the maximum gain should be at far elevation angles, considered at $\theta= \pm 60^{\circ}$ for the satellite at orbit far $800 \mathrm{~km}$ from the earth (Figure 6b). Then the constraints for this wide beam (Figure 7a) are as follows:

i. Position of the gain maxima for the Isoflux are at elevation angles $\theta= \pm 60^{\circ}$.

ii. $3 \mathrm{~dB}$ gain at $\theta= \pm 30^{\circ}$.

iii. The interesting zone for the Isoflux is bounded by $\left[\theta>30^{\circ}\right]$ and $\left[\theta<-30^{\circ}\right]$.

iv. Axial gain for the wide beam is not important, low gain in the axial direction considered between $\left[\begin{array}{lll}-30^{\circ} & 30^{\circ}\end{array}\right]$ elevation angles.

v. Axial ratio less than $3 \mathrm{~dB}$ in the regions of maximum Isoflux gain.

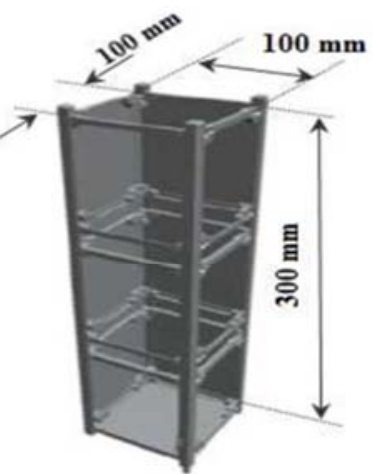

(a)

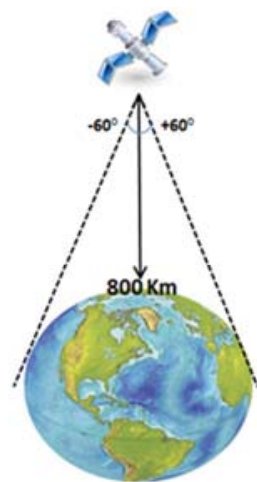

(b)

Figure 6. (a) Cube-sat $3 U$ platform, (b) LEO orbit to be launched on.

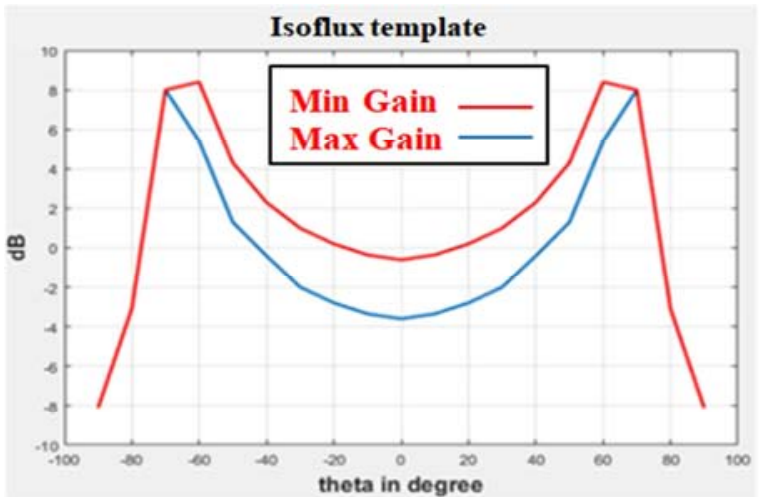

(a)

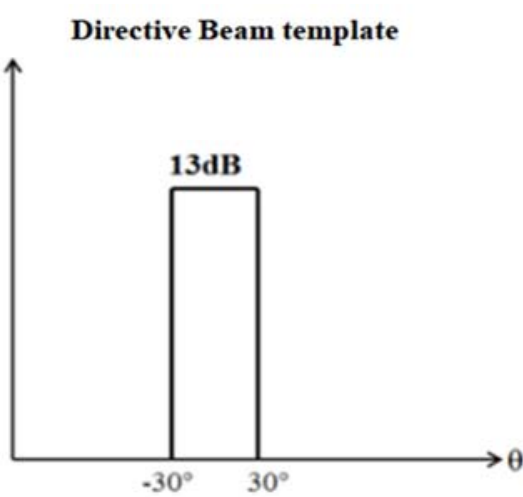

(b)

Figure 7. Two beams, (a) Isoflux mode template, (b) high gain axial mode. 
The second mode has $13 \mathrm{~dB}$ gain in the axial direction (Figure $7 \mathrm{~b}$ ) and the gain at $\theta= \pm 30^{\circ}$ must be greater than $3 \mathrm{~dB}$. From the two beams the switching elevation angles between the two modes are at $\theta= \pm 30^{\circ}$. This mode serves for high data rate link. Then the antenna will switch between the two modes according to the different CNES applications required, the switching angle is considered according the coverage area of each beam during the satellite-earth base stations communication. So, for the gain at this switching elevation angles in the two modes we have chosen to be $3 \mathrm{~dB}$ to avoid blind regions.

Throughout this review the manufacturing will concern the wide beam pattern, to show the advantages of using ARMA to achieve high gain at far elevation angles. Also the axial ratio for such wide beam will show the new electromagnetic approach enhancement to maintain the circular polarization inside the requirements. However the high axial gain mode will be under study and simulation only.

\section{Circularly Polarized Pixel}

ARMA approach shows big advantages to be used in comparison with the classical antenna arrays in linear polarization $[7,8]$; this made it possible to a major axis of research around antennas with radiation agility with circular polarization. Remind that the pixel design is the first step to do that. Then, the choice of the elementary source for circularly polarized ARMA system will be the main point to build such agile antennas. In our work the patch inside the EBG cavity is the source of the circularly polarized field. Many structures are possible to generate circular polarization including: truncated edges patch which is easy to design (low bandwidth around $2 \%$ and bad axial ratio in terms of wide elevation angles [11]), slotted patch, dual feed patch, and quad-feed patch. We are interested in our study about the multi feeds especially the quad-feed solution to be discussed

\section{Axial Ratio}

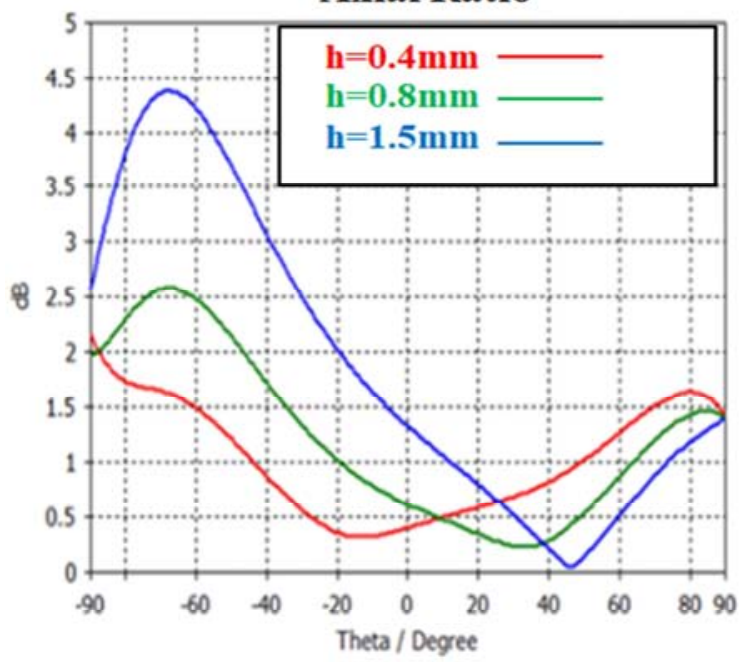

(a)

briefly.

The feeds (in the two feed structure) are phase shifted by $90^{\circ}$ in the case of two feeding probes (Figure $8 \mathrm{~b}$ ), where the uniform radiating surface is achieved (Figure $8 \mathrm{c}$ ). The axial ratio is below $3 \mathrm{~dB}$ for the elevation angles between $\pm 47^{\circ}$ (Figure 8e) which is not wide enough but could be suitable for some applications. Also the axial ratio and gain patterns are not completely symmetrical in all the azimuth planes (Figure 8d and Figure 8e).

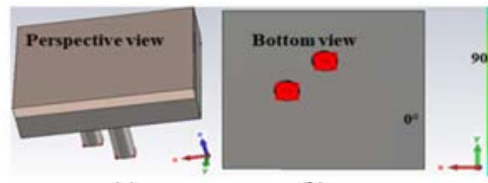

(a)

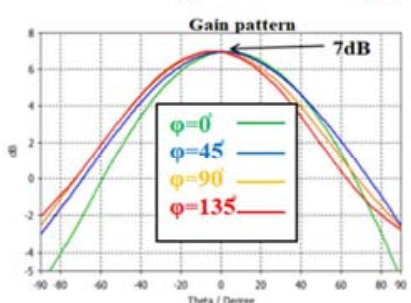

(d)

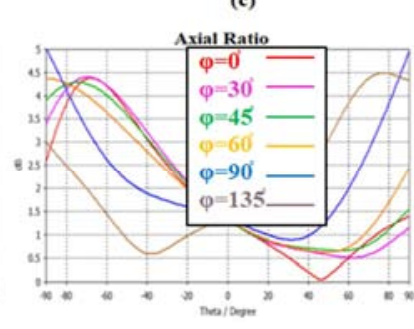

(e)
Figure 8. Pixel with two ports $(0.5 \lambda x 0.5 \lambda)$, (a) perspective view, (b) bottom view, (c) uniform radiating surface, (d) gain pattern at $8.2 \mathrm{GHz}$, (e) axial ratio pattern at $8.2 \mathrm{GHz}$.

For this design enhancement of the axial ratio was done by decreasing the substrate height of the patch (inside the cavity). In other world increasing the EBG cavity height shows good enhancement but also decreases the bandwidth. Figure 9 shows the axial ratio enhancement at different substrate heights $(0.4,0.8$ and $1.5 \mathrm{~mm})$ and bandwidth corresponds.

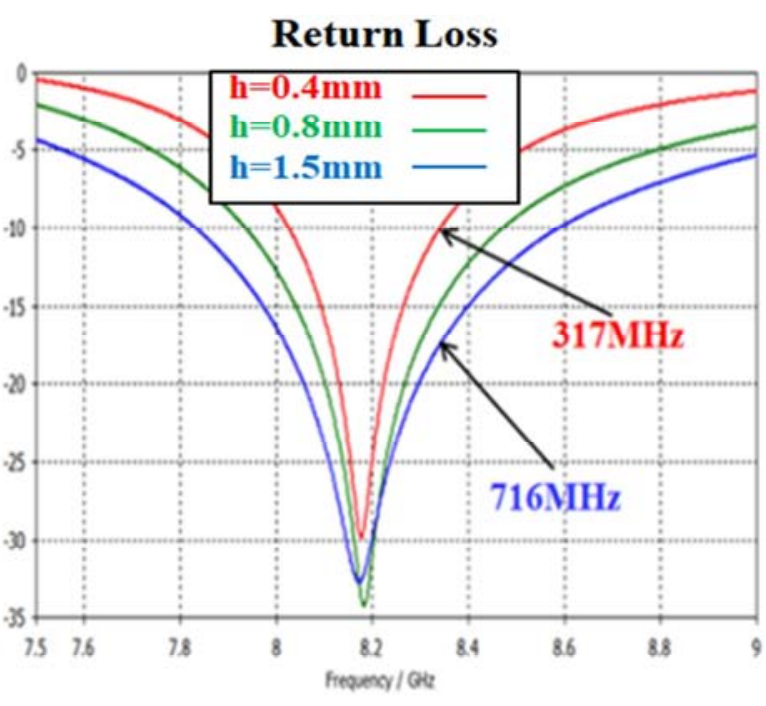

(b)

Figure 9. (a) Axial ratio enhancement, (b) bandwidth for substrate heights $0.4,0.8$ and $1.5 \mathrm{~mm}$. 
Looking for better solution we build a pixel with a patch fed by four ports with phase shifts of $0^{\circ}, 90^{\circ}, 180^{\circ}$ and $270^{\circ}$ (Figure 10). This pixel design will be the main step for building our circularly polarized matrix antenna. For that the performance of this pixel (four feedings) will be shown directly in comparison with the classical patch antenna used in AESA. Classical patch antenna is designed with same dimensions of the pixel $(0.5 \lambda \times 0.5 \lambda)$ with four feeding ports.

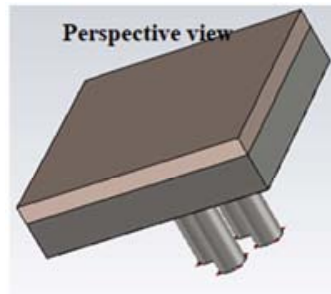

(a)
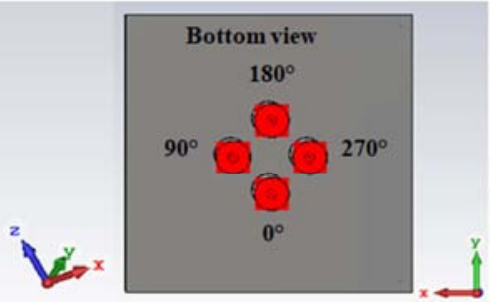

(b)

Figure 10. Pixel with four feeding ports, (a) perspective view, (b) bottom view.

Figure $11 \mathrm{~b}$ shows the uniformity of the radiating surface of the four ports pixel where it is not the case for the patch antenna (Figure 11a). That is the sampling required (quantification of the new approach). Figure 12 shows an improvement of the bandwidth for the pixel in comparison with the patch. Pixel bandwidth is $985 \mathrm{MHz}(12 \%)$ and patch bandwidth is $423 \mathrm{MHz}(5.1 \%)$, this high bandwidth of the pixel is due to the low profile EBG cavity [12]. This
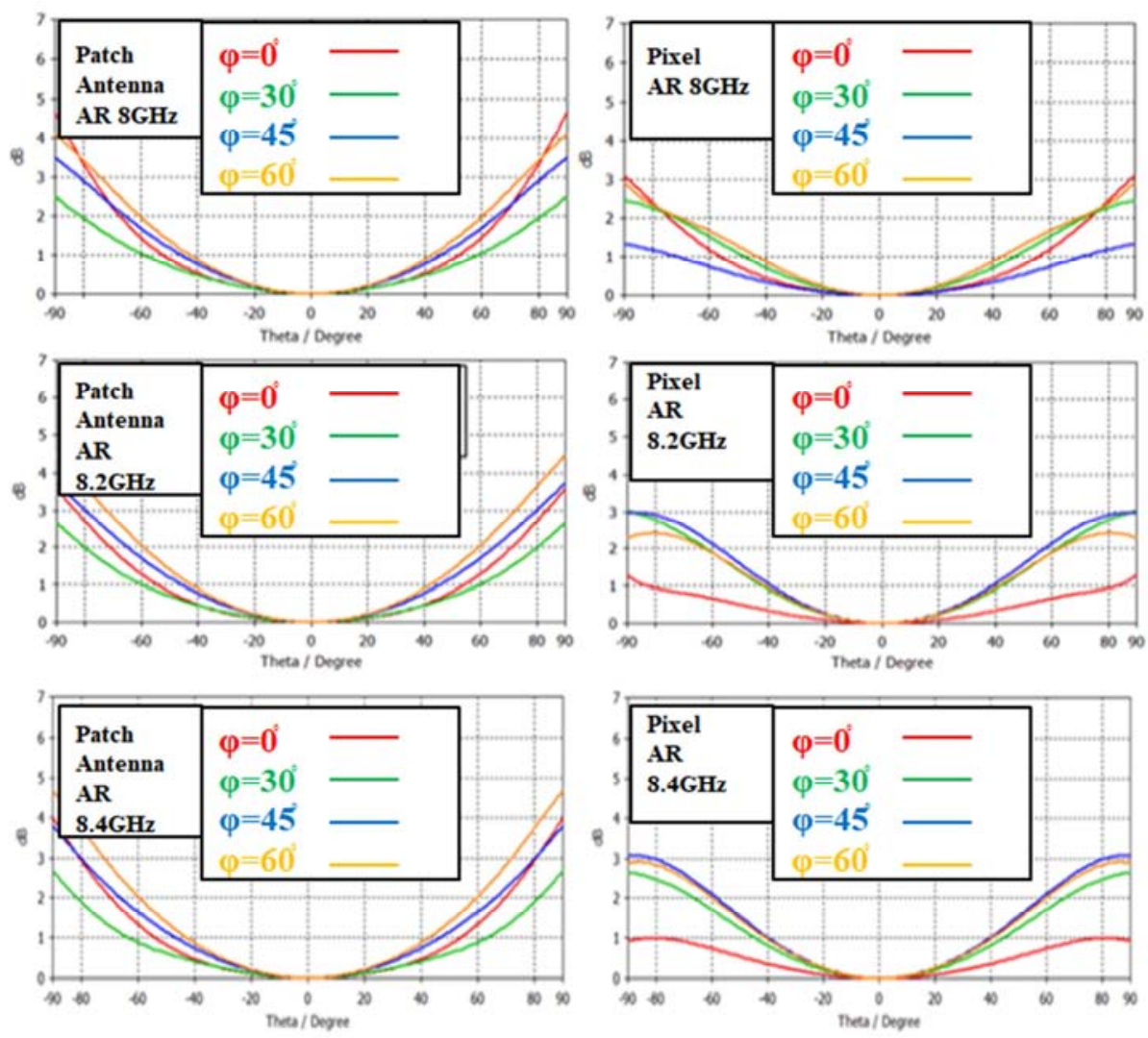

Figure 13. Axial ratio ( $f=8,8.2$ and $8.4 \mathrm{GHz})$, (left) classical patch, (right) pixel. 


\section{Polarization Circuits Design}

This CP elementary pixel must be fed by RF-circuit that insures the phase shifts at the pixel ports. Two circuits are designed one for the four ports pixel and one for the two ports case. The $90^{\circ}$ hybrid coupler is used since it has more output stability in magnitude and phase [13] and the circuits are printed on TMM10i substrate with height $0.381 \mathrm{~mm}$, $\varepsilon_{r}=9.8$ and $\tan \delta=0.002$.
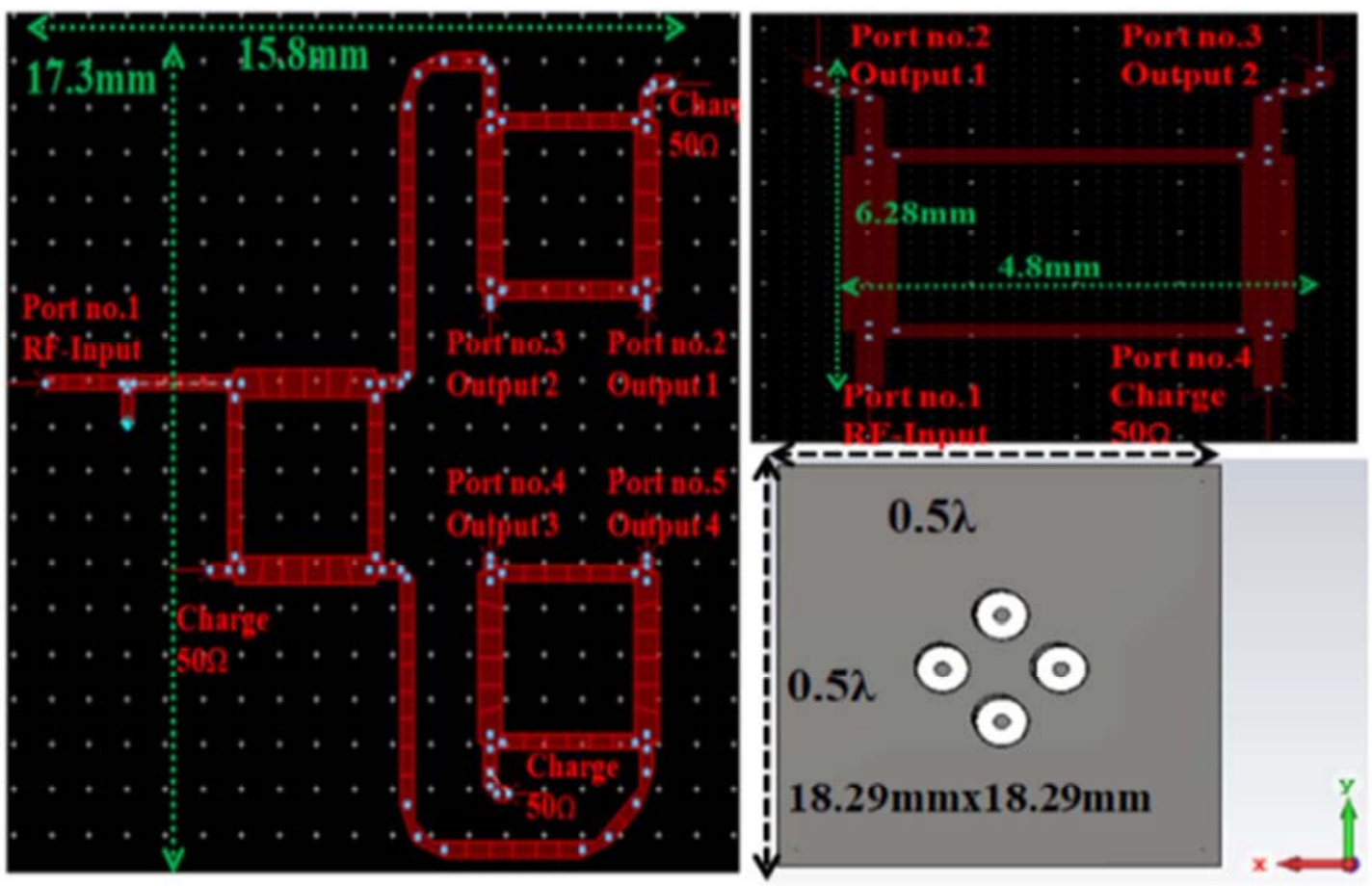

Figure 14. Circuits with two and four outputs and the limited aread behind the pixel.

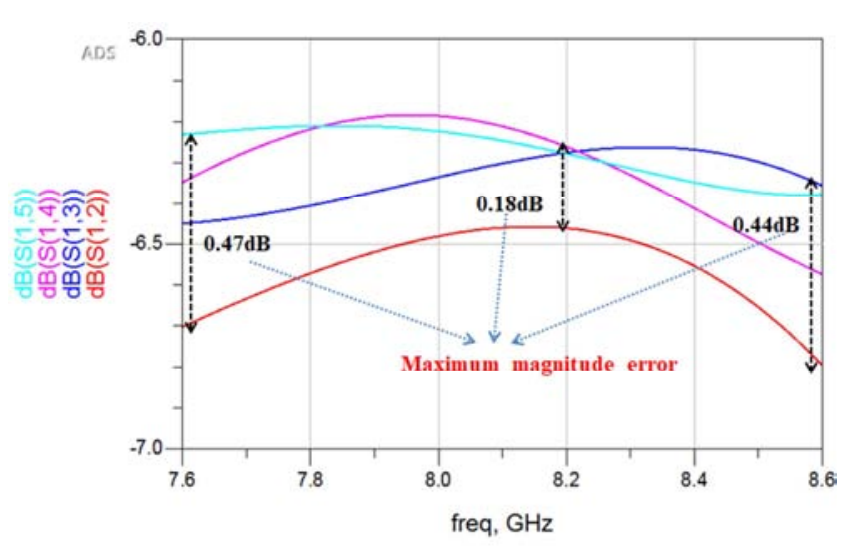

Figure 15. Output magnitude of the 4 ports circuit over $1 G H z$ band with maximum $0.47 \mathrm{~dB}$ error.

\section{Isoflux Beam Generation 1D Matrix Antenna}

This paragraph presents the design of $1 \mathrm{D}$ matrix antenna with the isoflux beam generation. The $2 \mathrm{D}$ design will be extended next to develop the $2 \mathrm{D}$ matrix antenna, where the
The two circuits are shown in Figure 14 with the main constraint in these designs which is the limited area allowed below the pixel $(18.29 \mathrm{~mm} \times 18.29 \mathrm{~mm}$ working at $8.2 \mathrm{GHz}$ central frequency). Magnitude outputs in the desired band are shown in Figure 15 showing the maximum corresponding errors (for the four outputs case). The maximum magnitude error is obtained when using four ports circuit is $0.47 \mathrm{~dB}$ and the maximum phase error is $6^{\circ}$. feeding law will be derived from the $1 \mathrm{D}$ to $2 \mathrm{D}$ case before doing optimizations. In the 1D solution AESA and ARMA will be compared in terms of gain at far elevation angles and the axial ratio to show one of the advantages of the new approach.

In this paragraph the two antennas ARMA and AESA will be used for beam forming, the beam considered is wellknown as "Isoflux" [14, 15] working in the X-band centered at $8.2 \mathrm{GHz}$. This type of radiation pattern is essential in many space applications for the earth observation and telecommunication between terrestrial and satellites data links. If we consider a satellite in the low earth orbit (LEO) far $800 \mathrm{~km}$ from the earth (Figure 6b), then the gain pattern "Isoflux" must follow special conditions with maximum radiation at far elevation angles $\pm 60^{\circ}$ and minimum in the axial direction (Figure $7 \mathrm{~b}$ shows the beam template), such that the power under the effect of many losses mainly the path loss, is received at the earth data links with equal power.

Formation of the 1D isoflux beam requires feeding AESA and ARMA with special law following the concept of the SFT that relates the radiating surface to the radiated far-field. To obtain the isoflux pattern an approximation was done, using the Sinc-shape for the radiating surface we obtain 
theoretically the rectangular radiation pattern, and then with the effects of the antenna edges, the reflection with the rectangular shape will give an isoflux pattern (Figure 16). So, the radiation surfaces of both ARMA and AESA are chosen to have "Sinc" shape, the Sinc is sampled and five samples are distributed as weights for the antennas. 1D $(1 \times 5)$ antennas are used with $0.5 \lambda$ periodicity (Figure 16).
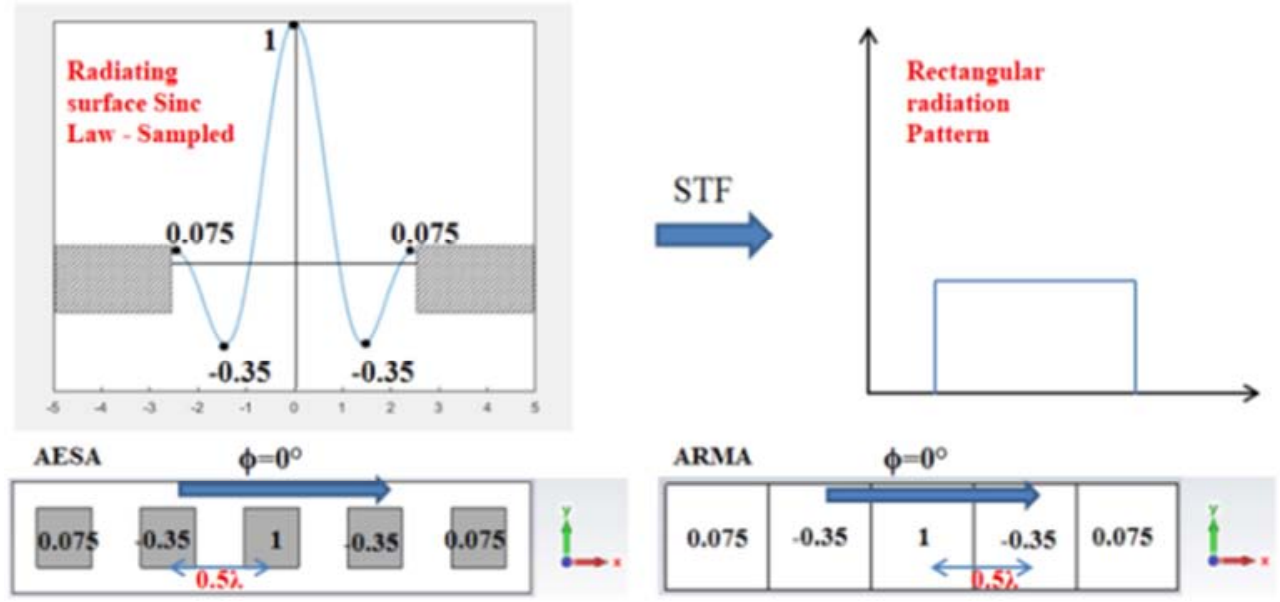

Figure 16. Feeding law for ARMA and AESA for $1 D$ Isoflux pattern.

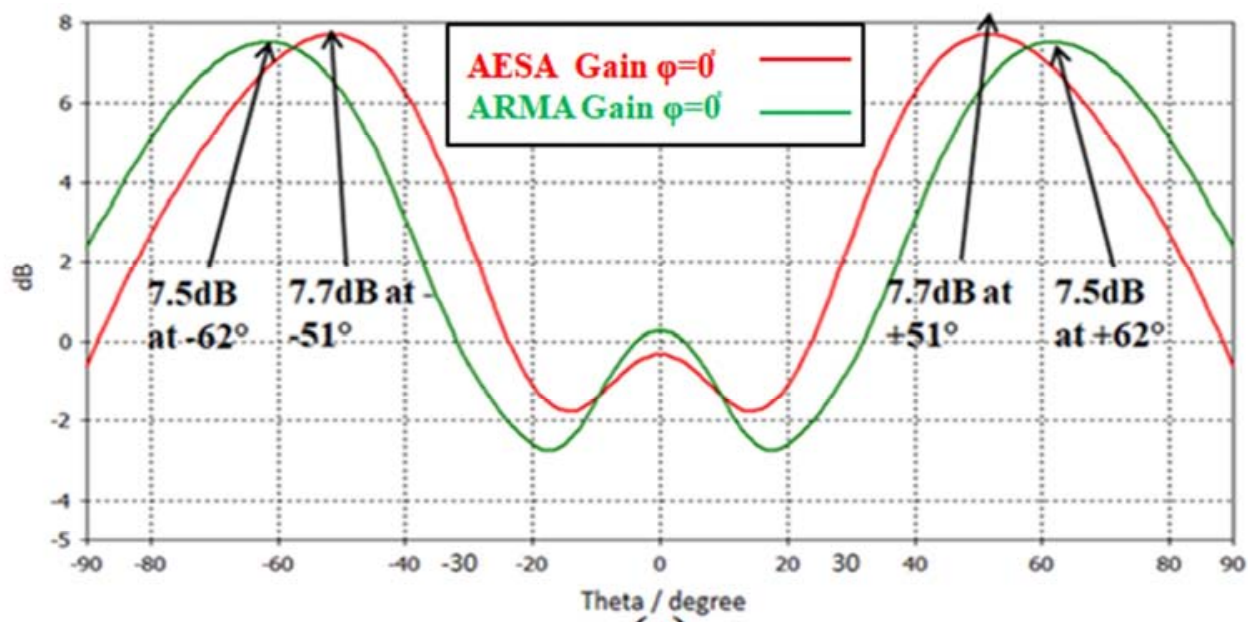

(a)

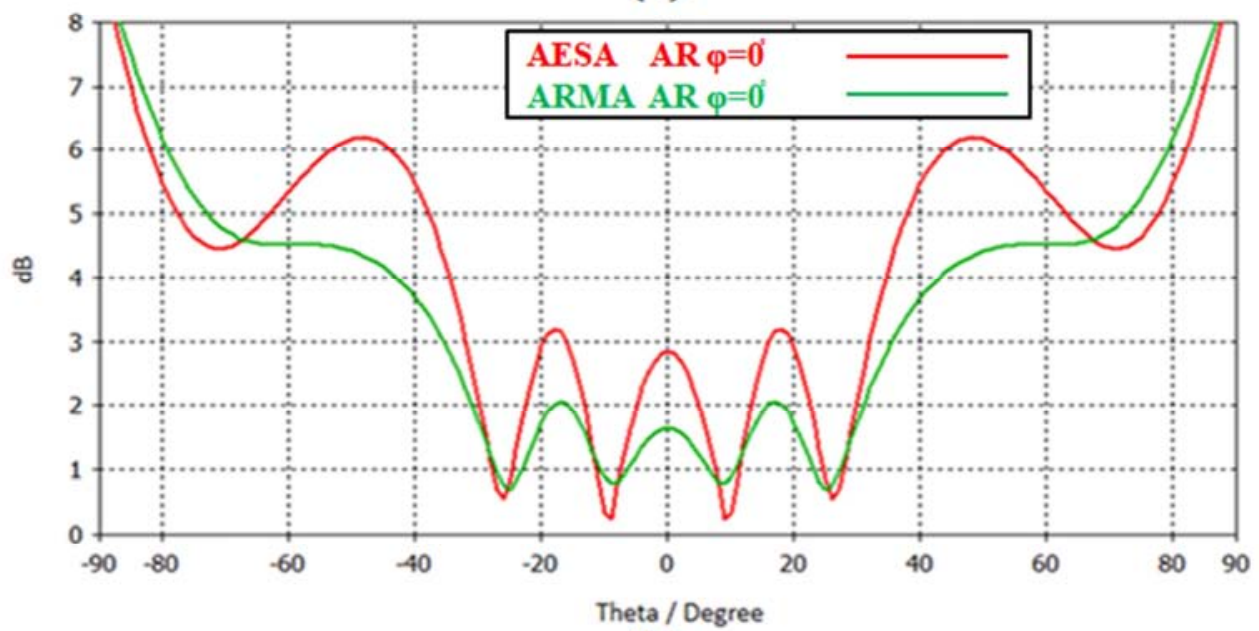

(b)

Figure 17. 1D Isoflux, (a) gain pattern comparison, (b) axial ratio comparison.

The results of the gain and axial ratio patterns of the two antennas ARMA and AESA are compared in Figure 17 for the azimuth plane $\varphi=0^{\circ}$ at $8.2 \mathrm{GHz}$. Both results of the gain follow the template given by Figure $17 \mathrm{~b}$, but the gain of 
AESA is slightly higher $(0.2 \mathrm{~dB}$ not very significant $)$; however the position of the maximum gain for the Isoflux in ARMA is better according to the template. In ARMA maximum gain is $7.5 \mathrm{~dB}$ at $\theta= \pm 61^{\circ}$ where in AESA the maximum gain is $7.7 \mathrm{~dB}$ at $\theta= \pm 50^{\circ}$ (Figure 17a). Also the axial ratio in ARMA is better (lower by $1 \mathrm{~dB}$ to $1.8 \mathrm{~dB}$ ), especially in the regions where the Isoflux starts to have maximum gain $\left(\left[-30^{\circ}-70^{\circ}\right]\right.$ and $\left[30^{\circ} 70^{\circ}\right]$ ) (Figure $\left.17 \mathrm{~b}\right)$.

\section{Circularly Polarized 2D ARMA Realization}

Two beams modes are required Isoflux and directive beams (Figure 7) in the different azimuth planes giving arise to the usage of the two dimensional ARMA.

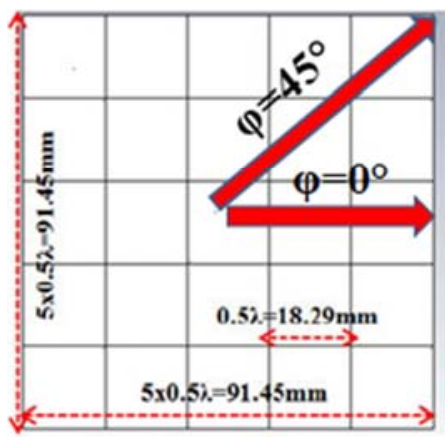

(a)

\subsection{Matrix Architecture}

The matrix antenna dimensions are limited by $9 \mathrm{cmx} 9 \mathrm{~cm}$ to fit the area allowed on the top of the Nano-Satellite with a maximum antenna height of $30 \mathrm{~mm}$. good approximation of the beam forming and the sampled radiating surface relation with the radiation pattern require large enough sampling (number of pixels). For the given design specifications the best is to choose the size of the matrix to be $5 \times 5$ pixels with $0.5 \lambda$ periodicity. Each pixel is fed by four ports for the aim of circular polarization. Matrix architecture is given in Figure 18 showing the dimensions $(91.45 \mathrm{~mm} \times 91.45 \mathrm{~mm})$ with height $3.1 \mathrm{~mm}$ without the circuit to be printed below and $3.481 \mathrm{~mm}$ with.

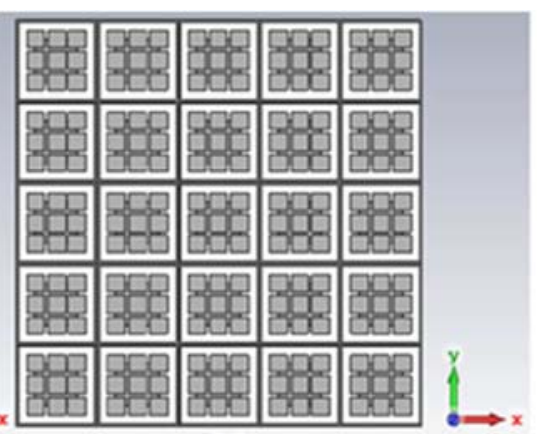

(b)

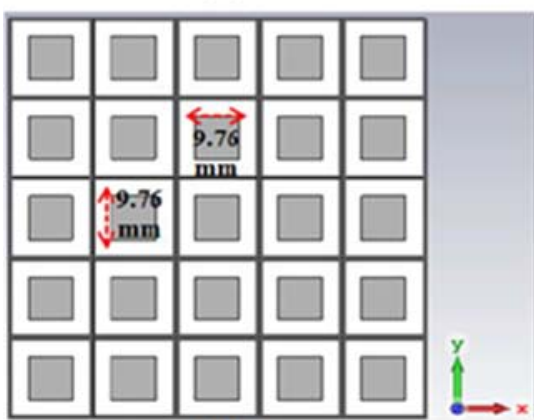

(c)

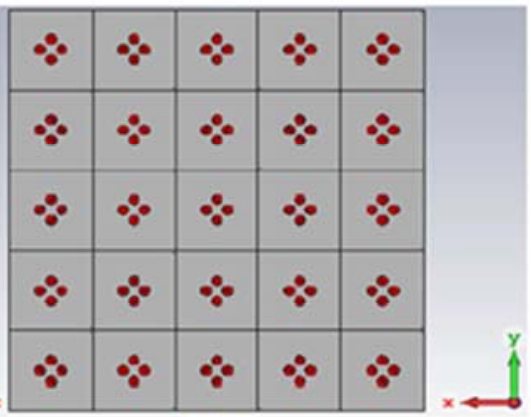

(d)

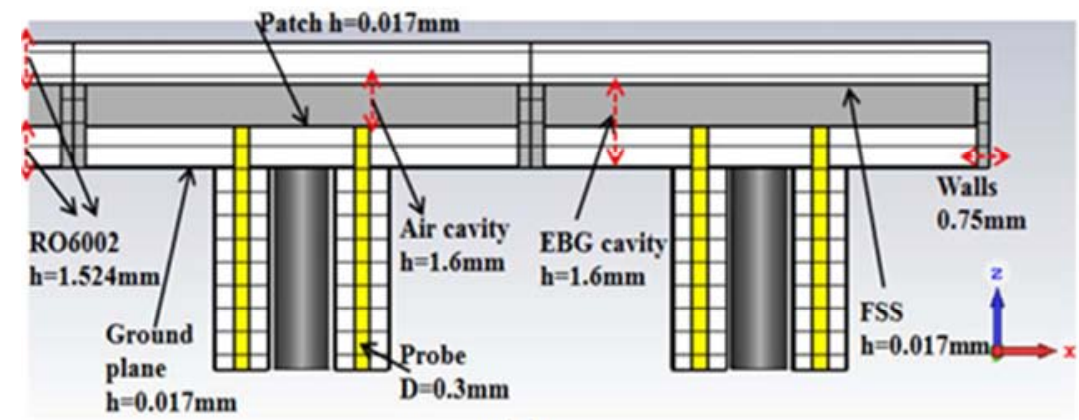

(e)

Figure 18. 2D ARMA, (a) top view, (b) top view showing FSS layer, (c) top view showing patch layer, (d) bottom view, (e) cut view.

\subsection{Isoflux Formation (2D) Using Butler Matrix and Feeding Law Optimization}

First method applied to generate the 2D feeding law is derived using the Butler matrix technique (Figure 19) from the 1D isoflux feeding law (Figure 16). The results of the Isoflux gain and the axial ratio patterns at $8.2 \mathrm{GHz}$ are shown in Figure 20; the gray color indicates the interesting region of the isoflux mode. The considered azimuth planes are $\varphi=0^{\circ}$, $22.5^{\circ}, 45^{\circ}, 67.5^{\circ}$ and $90^{\circ}$. The maximum of the isoflux gain 
is $4.72 \mathrm{~dB}$ located at $\theta= \pm 52^{\circ}$ in the azimuth plane $\varphi=45^{\circ}$, whereas it is not the case for the azimuth planes. The location of the maxima and its value varies in the other planes; this dissymmetry in the pattern is due to the reflections from the antenna edges that are not the same in all the directions due to square-shaped matrix (Figure 18a). The worst case of the pattern occurs in the plane $\varphi=45^{\circ}$ where the maximum gain is $3.3 \mathrm{~dB}$ at $\theta= \pm 40^{\circ}$. The axial ratio is below $3 \mathrm{~dB}$ with some oscillations above $3 \mathrm{~dB}$ at some elevation angles, this is due to the surface current that makes reflections at the edges of the antenna.

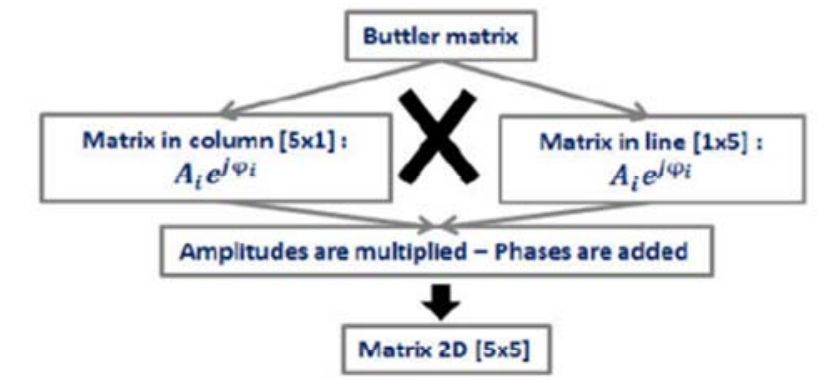

\begin{tabular}{|c|c|c|c|c|c|}
\hline \multicolumn{6}{|c|}{ Table of amplitudes $A_{i, j}$} \\
\hline & 1 & 2 & 3 & 4 & 5 \\
\hline 1 & 0 & 0,002 & 0,01 & 0.002 & 0 \\
\hline 2 & 0.002 & 0.03 & 0.18 & 0.03 & 0,002 \\
\hline 3 & 0.01 & 0.18 & 1 & 0.18 & 0.01 \\
\hline 4 & 0,002 & 0.03 & 0.18 & 0.03 & 0,002 \\
\hline 5 & 0 & 0.002 & 0.01 & 0.002 & 0 \\
\hline
\end{tabular}

\begin{tabular}{|c|c|c|c|c|c|}
\hline \multicolumn{7}{|c|}{ Table of $\varphi_{i, j}$} \\
\hline & 1 & 2 & 3 & 4 & 5 \\
\hline 1 & $0^{\circ}$ & $180^{\circ}$ & $0^{\circ}$ & $180^{\circ}$ & $0^{\circ}$ \\
\hline 2 & $180^{\circ}$ & $0^{\circ}$ & $180^{\circ}$ & $0^{\circ}$ & $180^{\circ}$ \\
\hline 3 & $0^{\circ}$ & $180^{\circ}$ & $0^{\circ}$ & $180^{\circ}$ & $0^{\circ}$ \\
\hline 4 & $180^{\circ}$ & $0^{\circ}$ & $180^{\circ}$ & $0^{\circ}$ & $180^{\circ}$ \\
\hline 5 & $0^{\circ}$ & $180^{\circ}$ & $0^{\circ}$ & $180^{\circ}$ & $0^{\circ}$ \\
\hline
\end{tabular}

Figure 19. 2D ARMA feeding law for Isoflux pattern (Butler Matrix).

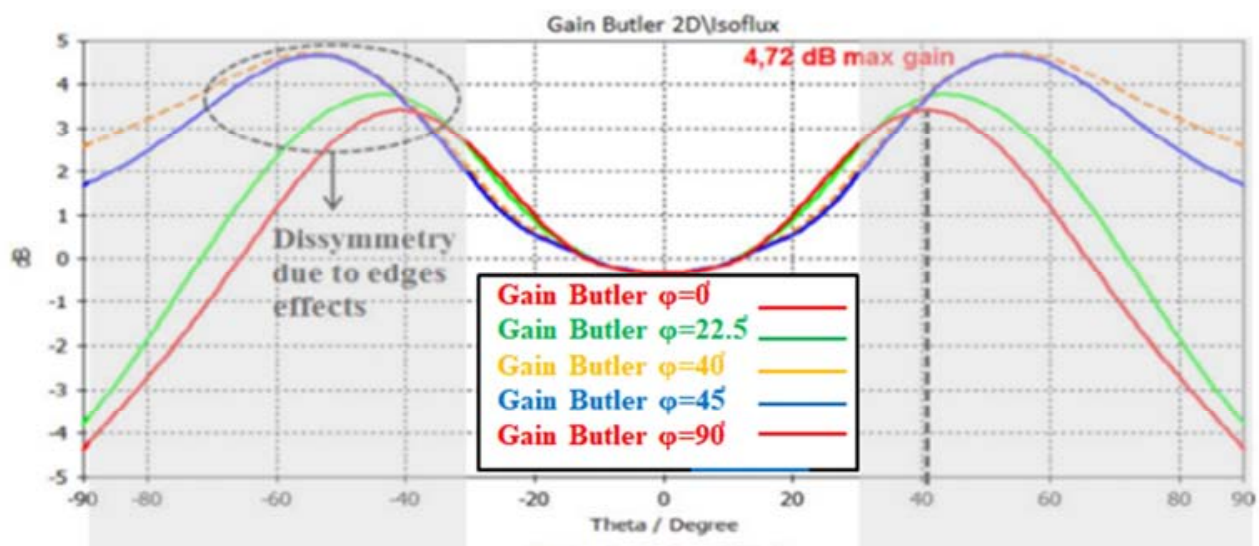

Axial ratio Butler 201isoflux

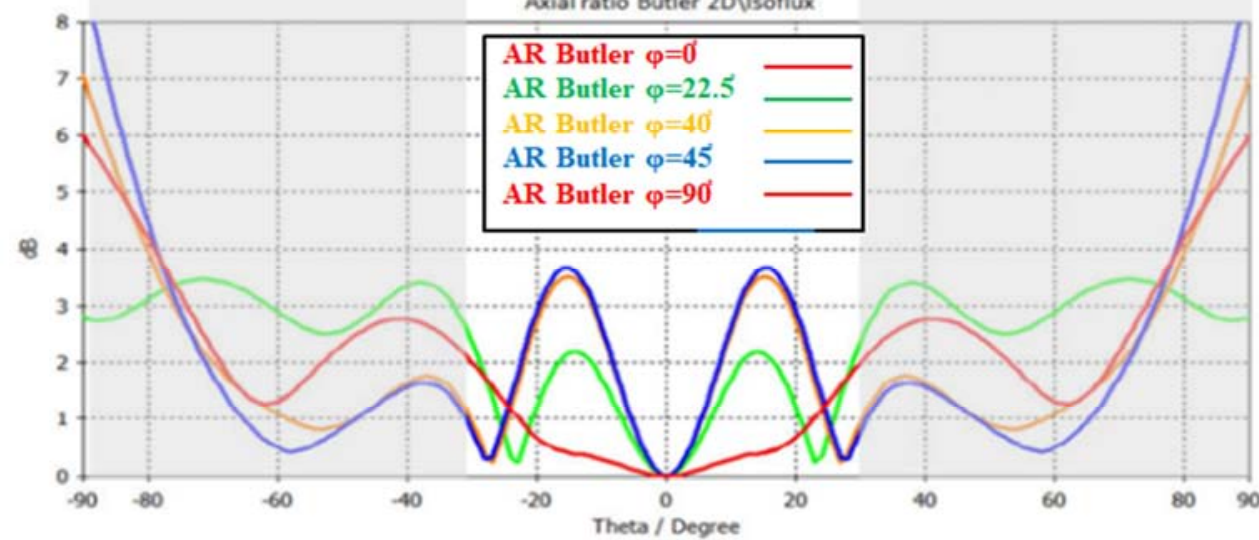

Figure 20. Isoflux pattern using Butler method, (top) gain pattern, (bottom) axial ratio. 


\subsection{D Isoflux Feeding Law Optimization}

\subsubsection{Using Synthesis Algorithm}

The feeding law is optimized using synthesis algorithm. This synthesis algorithm depends on Min-Max search of the weights by several iterations to find the optimal solution (using the template given in Figure 7a). This algorithm was developed in XLIM Limoges University working on antenna arrays and beam forming. The weights resulted from this solution have a Sinc shape in all the directions of the antenna, that is why it is called Sinc-Circular. Feeding law distributed on the $5 \times 5$ ARMA is shown in Figure 21 . Compared to the Butler solution the Sinc-Circular is better regarding the Isoflux maximum gain locations, its wider by $3^{\circ}$ (example shown in Figure 21 for the azimuth plane $\varphi^{\circ} 0^{\circ}$ ). Also the axial ratio of the Sinc-circular solution is better especially at the far elevation angles $\left[-90^{\circ}\right.$ to $\left.-40^{\circ}\right]$ and $\left[40^{\circ}\right.$ to $\left.90^{\circ}\right]$ (Figure 21).

\begin{tabular}{|c|c|c|c|c|c|}
\hline \multicolumn{6}{|c|}{ Table of amplitudes $A_{i, j}$} \\
\hline & 1 & 2 & 3 & 4 & 5 \\
\hline 1 & 0 & 0,002 & 0,01 & 0.002 & 0 \\
\hline 2 & 0.002 & 0.03 & 0.13 & 0.03 & 0,002 \\
\hline 3 & 0.01 & 0.13 & 1 & 0.13 & 0.01 \\
\hline 4 & 0,002 & 0.03 & 0.13 & 0.03 & 0,002 \\
\hline 5 & 0 & 0.002 & 0.01 & 0.002 & 0 \\
\hline
\end{tabular}

\begin{tabular}{|c|c|c|c|c|c|}
\hline \multicolumn{7}{|c|}{ Table of $\varphi_{i, j}$} \\
\hline & 1 & 2 & 3 & 4 & 5 \\
\hline 1 & $0^{\circ}$ & $0^{\circ}$ & $0^{\circ}$ & $0^{\circ}$ & $0^{\circ}$ \\
\hline 2 & $0^{\circ}$ & $180^{\circ}$ & $180^{\circ}$ & $180^{\circ}$ & $0^{\circ}$ \\
\hline 3 & $0^{\circ}$ & $180^{\circ}$ & $0^{\circ}$ & $180^{\circ}$ & $0^{\circ}$ \\
\hline 4 & $0^{\circ}$ & $180^{\circ}$ & $180^{\circ}$ & $180^{\circ}$ & $0^{\circ}$ \\
\hline 5 & $0^{\circ}$ & $0^{\circ}$ & $0^{\circ}$ & $0^{\circ}$ & $0^{\circ}$ \\
\hline
\end{tabular}
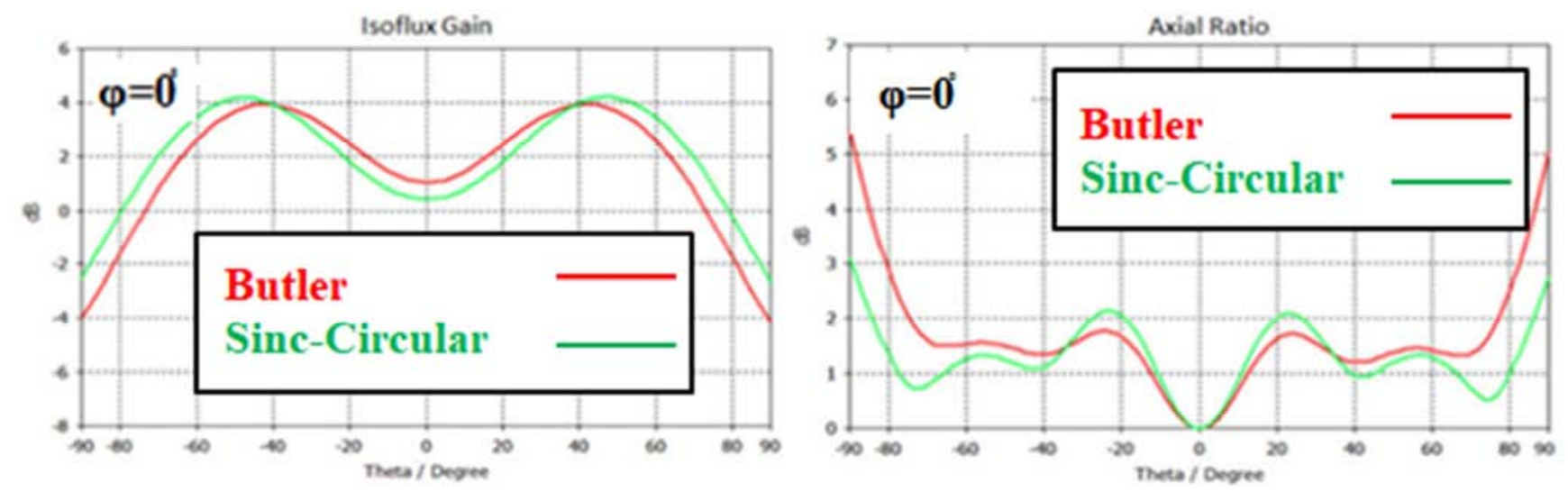

Figure 21. Isoflux pattern enhancement using Sin-Circular feeding law.

\subsubsection{Study of Peripheral Pixels}

For the aim of easier realization of the power divider it is of great interest for such design to be with lower number of outputs. For that the effect of the weights of the feeding law at the peripheral pixels of the matrix are studied. These weights are very small compared with the others and eliminating them (set to zero in simulation and connected to $50 \mathrm{ohm}$ charge in realization) has no significant effects on the antenna patterns. This is demonstrated and verified in Figure 22 (example of gain pattern $\varphi^{\prime} 0^{\circ}$ ). In this way the power divider to be designed is a one input nine outputs instead of

25 outputs. This feeding law solution is called Sinc-Circular zero edges.

However eliminating these pixels at the peripherals in the realization $(3 \times 3$ matrix instead of $5 \times 5)$ has negative drawbacks on the axial ratio. The reflections from the nearer edges will highly degrade the axial ratio $(10 \mathrm{~dB}$ at some elevation angles). That is why using of $5 \times 5$ matrix and forcing the peripheral pixel as zero weighted is recommended. Results of this study are illustrated by an example of the axial ratio for the azimuth plane $\varphi=45^{\circ}$ in Figure 22. 


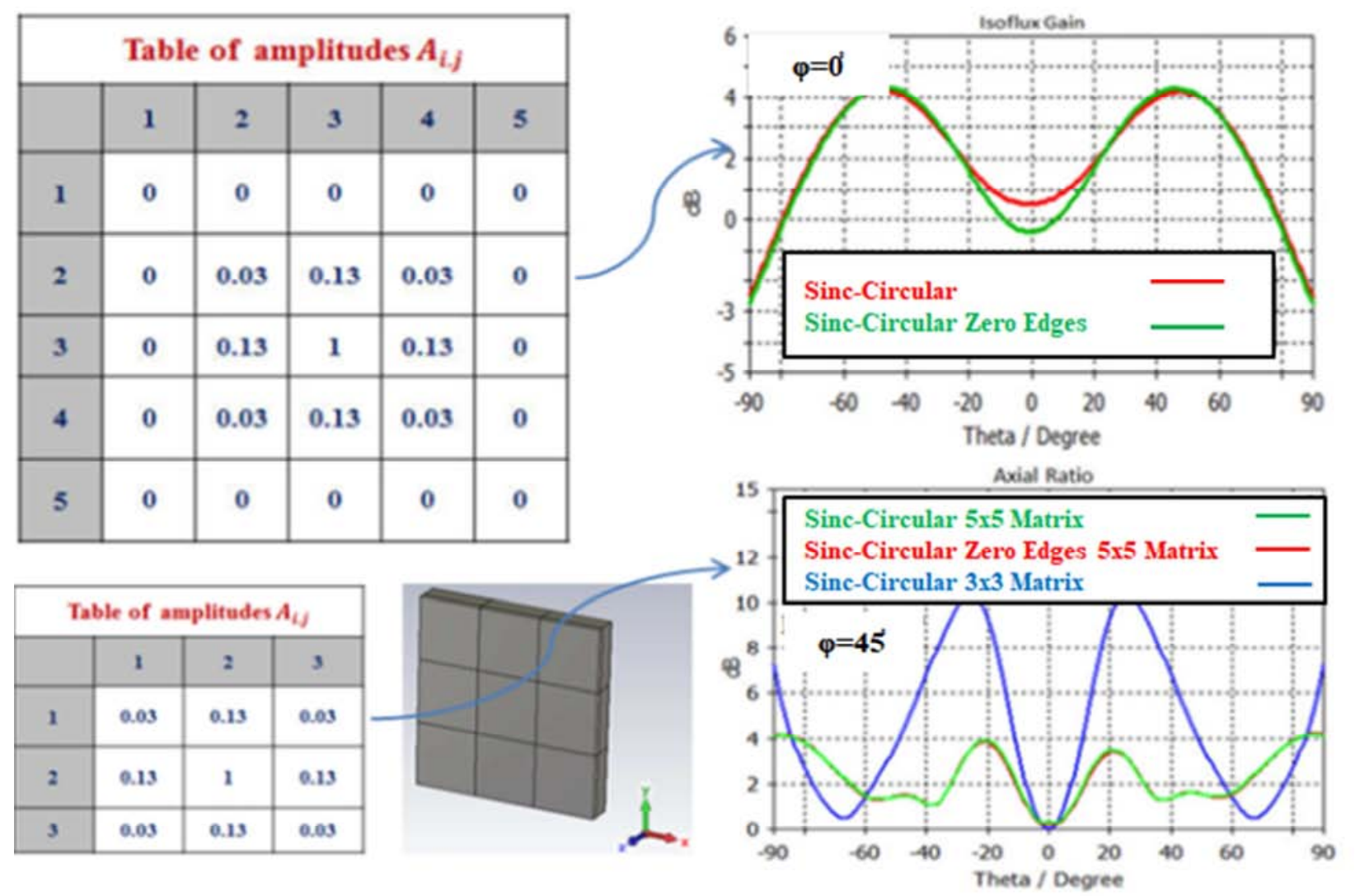

Figure 22. Feeding law optimization, (top) Sinc-Circular zero edges with corresponding effect, (bottom) $3 \times 3$ matrix antenna with axial ratio degradation.

\subsubsection{Optimization for Maximum Gain at Wider Azimuth Angles}

Several optimizations were done on the feeding law to get better isoflux maximum position and overcome the problem of the dissymmetry in the gain pattern in different the azimuth planes. This dissymmetry is obvious due to the unsymmetrical matrix design in all directions (square shape). The candidate weights under study are labeled " $\mathrm{C}$ stands for corner" and "M stands for middle" (Figure 23). These weights are studied using CST software analysis method, the best solution after several optimizations is obtained for $\mathrm{C}=\mathrm{M}=0.15$. This solution is based on Sin-Circular zero edges symmetrical solution that uses the same weights for the pixels around the central one. Also using such a solution makes easier the design of the nine output power divider.
Eight output weights are equally ( 0.15 compared to central pixel magnitude considered 1) with a phase shift of $180^{\circ}$ for eight outputs in terms of the central output phase.

Gain and axial ratio patterns for this solution are presented in Figure 23. The position of the maximum gain is not attained at $\theta= \pm 60^{\circ}$, but the gain at these angles $\left(\theta= \pm 60^{\circ}\right)$ is decreased no more than $1 \mathrm{~dB}$ from the maximum gain in each azimuth plane considered in the curves of Figure 23. The $3 \mathrm{~dB}$ switching gain position (between the bi-mode described) is at $\theta= \pm 33^{\circ}$ and not $\theta= \pm 30^{\circ}$ but this condition is not very significant according to the CNES applications requirements. The axial ratio is very good lower than $3 \mathrm{~dB}$ and fits the CNES specifications even if it is very high in some regions where the isoflux gain is very low and not in interest.

\begin{tabular}{|c|c|c|c|c|c|}
\hline \multicolumn{6}{|c|}{ Table of amplitudes $A_{i j}$} \\
\hline & 1 & 2 & 3 & 4 & 5 \\
\hline 1 & 0 & 0 & 0 & 0 & 0 \\
\hline 2 & 0 & $\mathrm{C}$ & $\mathrm{M}$ & $\mathrm{C}$ & 0 \\
\hline 3 & 0 & $\mathrm{M}$ & 1 & $\mathrm{M}$ & 0 \\
\hline 4 & 0 & $\mathrm{C}$ & $\mathrm{M}$ & $\mathrm{C}$ & 0 \\
\hline 5 & 0 & 0 & 0 & 0 & 0 \\
\hline
\end{tabular}

\begin{tabular}{|c|c|c|c|c|c|}
\hline \multicolumn{7}{|c|}{ Table of phases $\varphi_{i j}$} \\
\hline & 1 & 2 & 3 & 4 & 5 \\
\hline 1 & $0^{\circ}$ & $0^{\circ}$ & $0^{\circ}$ & $0^{\circ}$ & $0^{\circ}$ \\
\hline 2 & $0^{\circ}$ & $180^{\circ}$ & $180^{\circ}$ & $180^{\circ}$ & $0^{\circ}$ \\
\hline 3 & $0^{\circ}$ & $180^{\circ}$ & $0^{\circ}$ & $180^{\circ}$ & $0^{\circ}$ \\
\hline 4 & $0^{\circ}$ & $180^{\circ}$ & $180^{\circ}$ & $180^{\circ}$ & $0^{\circ}$ \\
\hline 5 & $0^{\circ}$ & $0^{\circ}$ & $0^{\circ}$ & $0^{\circ}$ & $0^{\circ}$ \\
\hline
\end{tabular}



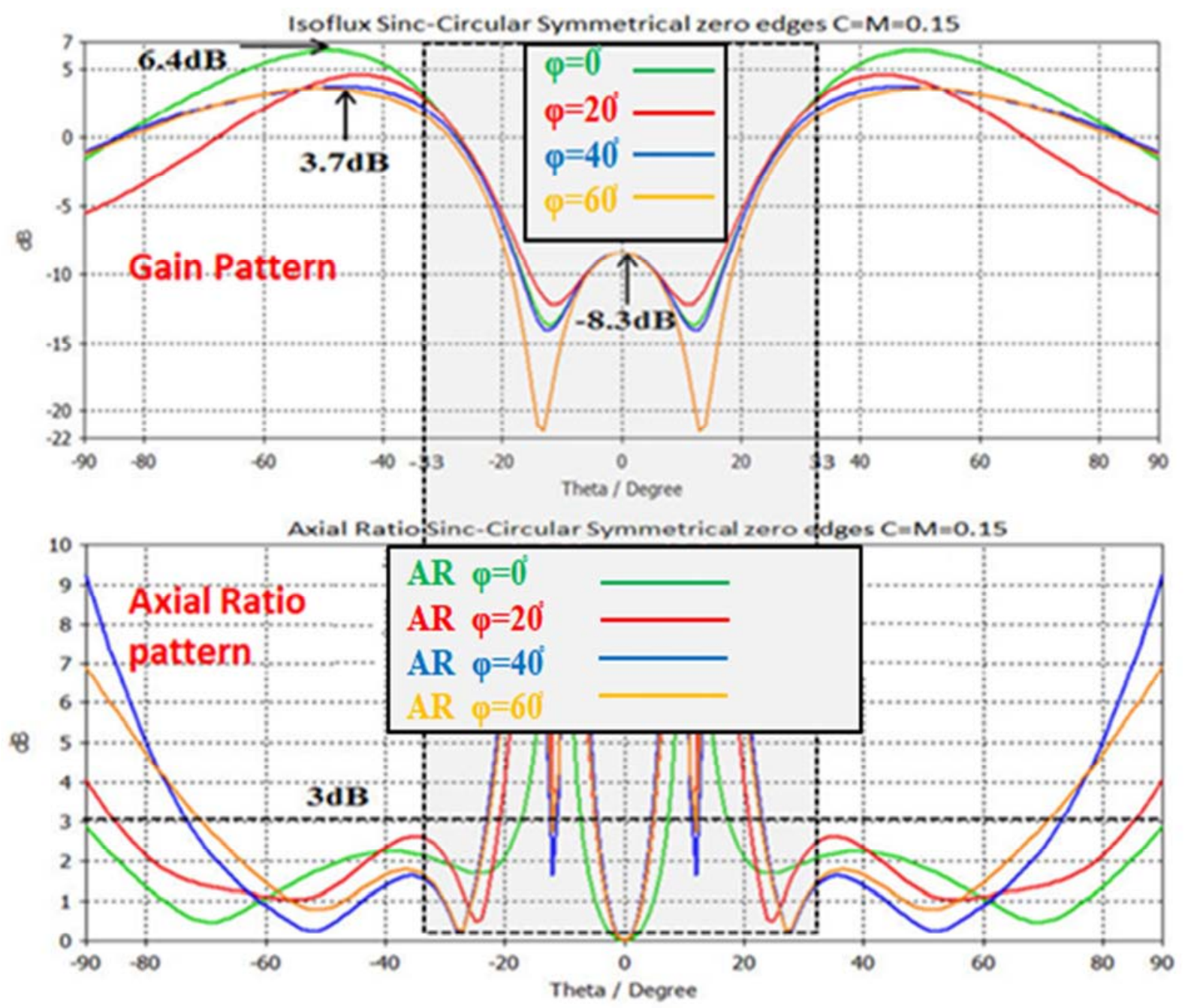

Figure 23. Symmetrical solution with $C=M=0.15$ and the corresponding gain and axial ratio patterns.

\subsection{Bimodal Beam Forming}

The axial beam requested by the CNES must have a maximum gain of $13 \mathrm{~dB}$, to do that nine pixels of the matrix are fed by equal weights (magnitudes and phases) with zeros at the matrix edges (Figure 24). The maximum gain obtained by this configuration is $14.2 \mathrm{~dB}$ with a gain $3 \mathrm{~dB}$ at the elevation angles $\theta= \pm 33^{\circ}$ and the axial ratio is below $3 \mathrm{~dB}$ is the entire interesting axial zone $\theta=\left[-30^{\circ}, 30^{\circ}\right]$ (Figure 24). The antenna can give a higher gain $19 \mathrm{~dB}$, this high gain is

\begin{tabular}{|l|l|l|l|l|}
\hline$\left(0,0^{\circ}\right)$ & $\left(0,0^{7}\right)$ & $\left(0,0^{7}\right)$ & $\left(0,0^{\circ}\right)$ & $\left(0,0^{7}\right)$ \\
\hline$\left(0,0^{7}\right)$ & $\left.0,0^{7}\right)$ & $\left.0,0^{7}\right)$ & $\left.0,0^{7}\right)$ & $\left(0,0^{7}\right)$ \\
\hline$\left(0,0^{\circ}\right)$ & $\left.0,0^{7}\right)$ & $\left.0,0^{7}\right)$ & $\left.0,0^{7}\right)$ & $\left(0,0^{\circ}\right)$ \\
\hline$\left(0,0^{7}\right)$ & $\left.0,0^{7}\right)$ & $\left.0,0^{7}\right)$ & $\left.0,0^{7}\right)$ & $\left(0,0^{7}\right)$ \\
\hline$\left(0,0^{\circ}\right)$ & $\left(0,0^{7}\right)$ & $\left(0,0^{7}\right)$ & $\left(0,0^{7}\right)$ & $\left(0,0^{7}\right)$ \\
\hline
\end{tabular}

not requested in the CNES specifications but it's presented to show the maximum gain the antenna can achieve. The $19 \mathrm{~dB}$ gain is achieved by feeding all the pixels of the $5 \times 5$ matrix as with equal weights, the axial ratio also below $3 \mathrm{~dB}$ shown in the aperture angle of the $19 \mathrm{~dB}$ beam (Figure 24). Several configurations were studied also to generate different types of axial beams; Sectorial one and axial isoflux but in the entire interesting axial range.

\begin{tabular}{|c|c|c|c|c|}
\hline$\left(0,0^{\circ}\right)$ & $\left(0,0^{\circ}\right)$ & $\left(0,0^{\circ}\right)$ & $\left.0,0^{\circ}\right)$ & $\left(0,0^{\circ}\right)$ \\
\hline$\left(0,0^{\circ}\right)$ & $\left(0,0^{+}\right)$ & $\left(0,0^{7}\right)$ & $\left(0,0^{\circ}\right)$ & $\left(0,0^{\circ}\right)$ \\
\hline$\left(0,0^{\circ}\right)$ & $\left(0,0^{\circ}\right)$ & $\left(0,0^{\circ}\right)$ & 0,09 & $\left(0,0^{\circ}\right)$ \\
\hline$\left(0,0^{\circ}\right)$ & $\left(0,0^{\circ}\right)$ & $\left.a, 0^{\prime}\right)$ & $\left(0,0^{\circ}\right)$ & $\left(0,0^{\circ}\right)$ \\
\hline$\left(1,0^{\circ}\right)$ & $\left(0,0^{\circ}\right)$ & $\left(0,0^{\prime}\right)$ & $(0,0)$ & $\left(0,0^{\circ}\right)$ \\
\hline
\end{tabular}




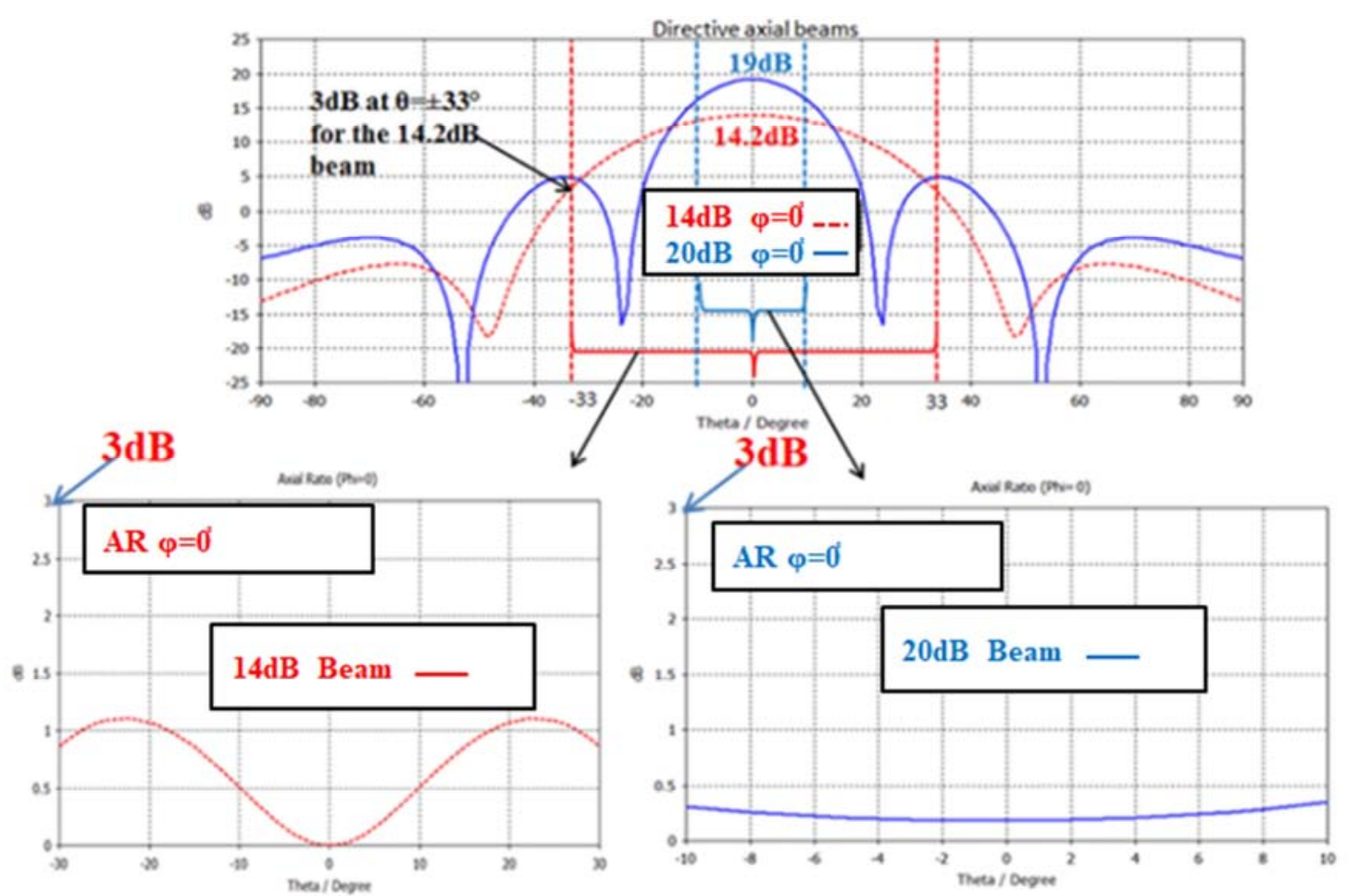

Figure 24. Axial Beam, (top) feeding law $3 \times 3$ and $5 \times 5$ weights, (blue), $19 \mathrm{~dB}$ gain with the axial ratio, (red) $14.2 \mathrm{~dB}$ gain with the axial ratio.

Figure 25 shows the two different patterns where the blue color and the grey color cover the interesting zones of the axial and wide isoflux beams respectively (azimuth planes $\varphi=0^{\circ}$ and $\left.\varphi=20^{\circ}\right)$. The wide isoflux beam corresponds for the symmetrical Sinc-Circular solution $(\mathrm{C}=\mathrm{M}=0.15$ zero edges $)$ studied previously. The curves show the two gain patterns with the corresponding axial ratio for each zone and the switching elevation angles between the two modes which is maintained at $\theta= \pm 30^{\circ}$.
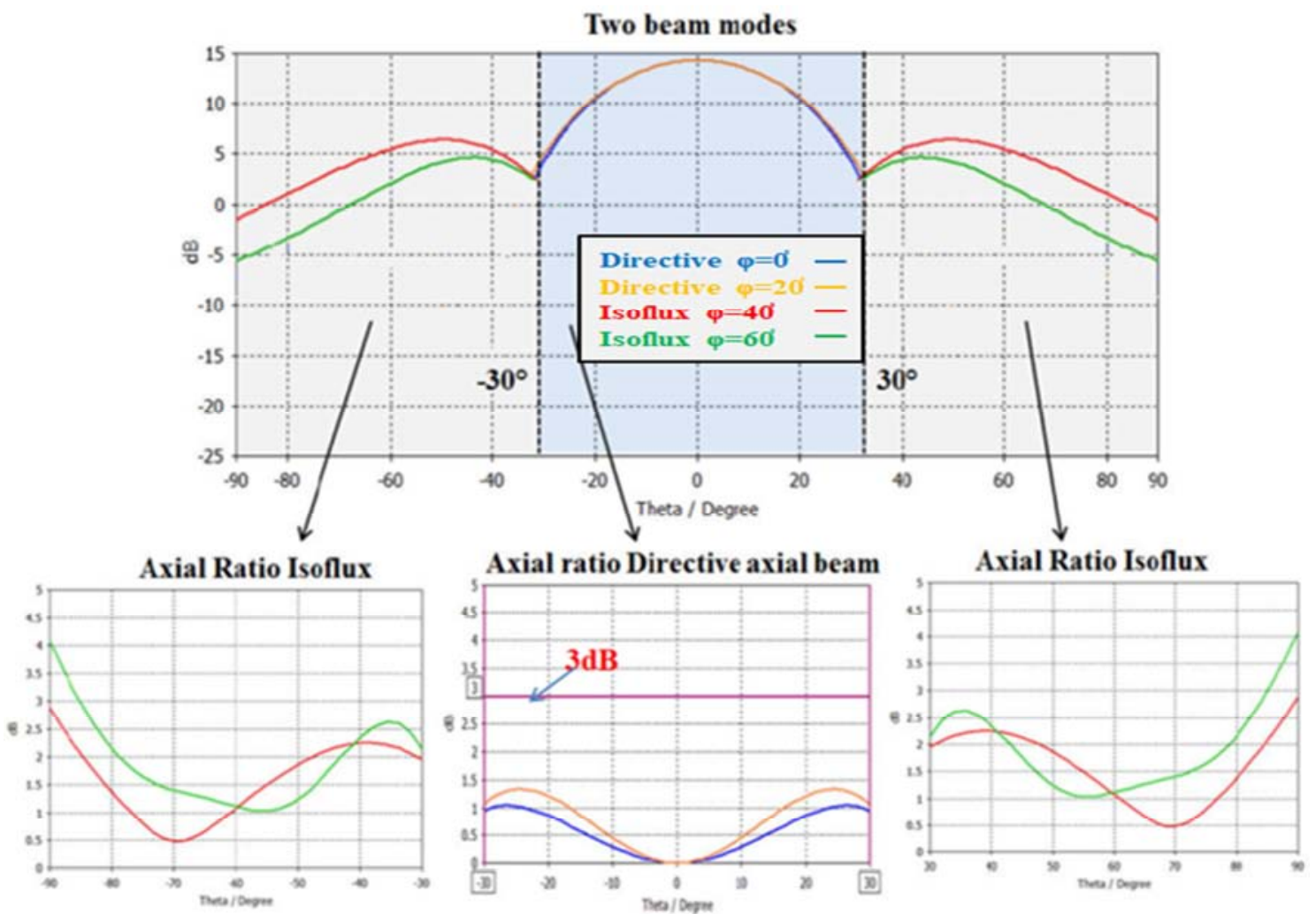

Figure 25. Two beams modes with the axial ratio $\varphi=0^{\circ}$ and $\varphi=20^{\circ}$. 


\section{Matrix Antenna Prototype Realization}

\subsection{Power Divider Realization}

To measure the directivity, gain, axial ratio and efficiency, we use an experimental device that realizes a power divider with 9 active outputs associated with a table of weights given in Figure 26b with $\mathrm{C}=\mathrm{M}=0.15$ and their corresponding phase is $180^{\circ}$ w.r.t the central phase $\left(0^{\circ}\right)$. This device is built with "commercial off the shelf components" (COTS) [16]. It is made of an equal amplitude and equal phase power divider with 18 ways. Only 9 outputs are used and the other ones are connected on $50 \Omega$ loads, as shown in Figure 26a.

The 8 ports corresponding to the pixels surrounding the central one are connected to attenuators to obtain an output level ratio close to 0.15 . The central pixel in the matrix antenna is fed by the central port called P6 through a phase shifter to obtain a $180^{\circ}$ phase shift with the surrounding pixel connected to other ports (P2, P3). The weights obtained experimentally with the device is presented in Figure 26b and compared with the theoretical one. The ratio between the central port and the other ones is slightly different $(0.17)$ due to the COTS attenuators resolution.

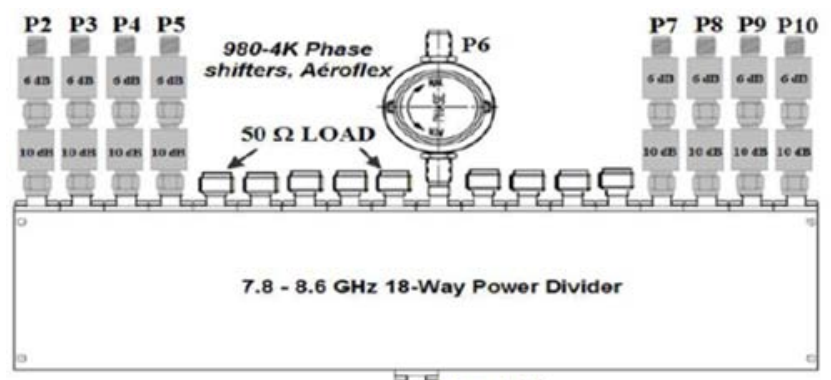

(a) Input P1

THE WEIGHTING FUNCTION

\begin{tabular}{|lcc|}
\hline & Ideal case & Experimental device \\
\hline Central pixel level & 1.00 & 0.17 \\
Other pixels level & 0.15 & 0.03 \\
linear ratio & 0.15 & 0.17 \\
Attenuation (dB) & -16.5 & -15.2 \\
\hline
\end{tabular}

(b)

Figure 26. Power divider, (a) CTOS experimental design, (b) measured weights compared to theoretical.

\subsection{ARMA Realization}

The matrix antenna is a multilayer design with FSS layer printed on RO6002 on the top, patch layer printed on RO6002 and polarization circuit's layer printed on TMM10i on the bottom [16]. The layers designed on CST are shown in Figure 27 assembled together with the metallic walls by rigid plastic screws.

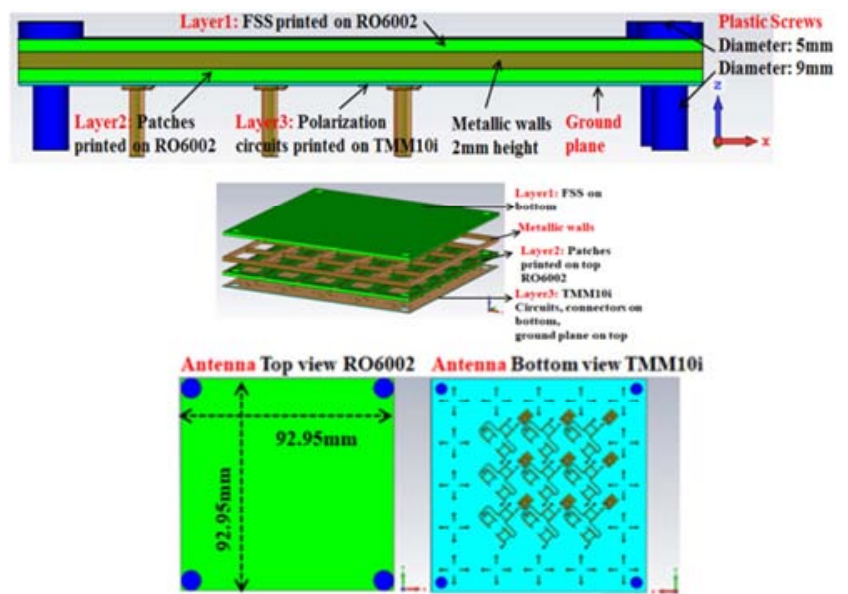

Figure 27. Matrix layers design (CST).

These layers are manufactured as shown in Figure 28a then assembled together and the return loss parameters and couplings (at the input of the circuits printed on the bottom Figure 28b ports P1 to P9) are measured using the VNA (vector network analyzer).
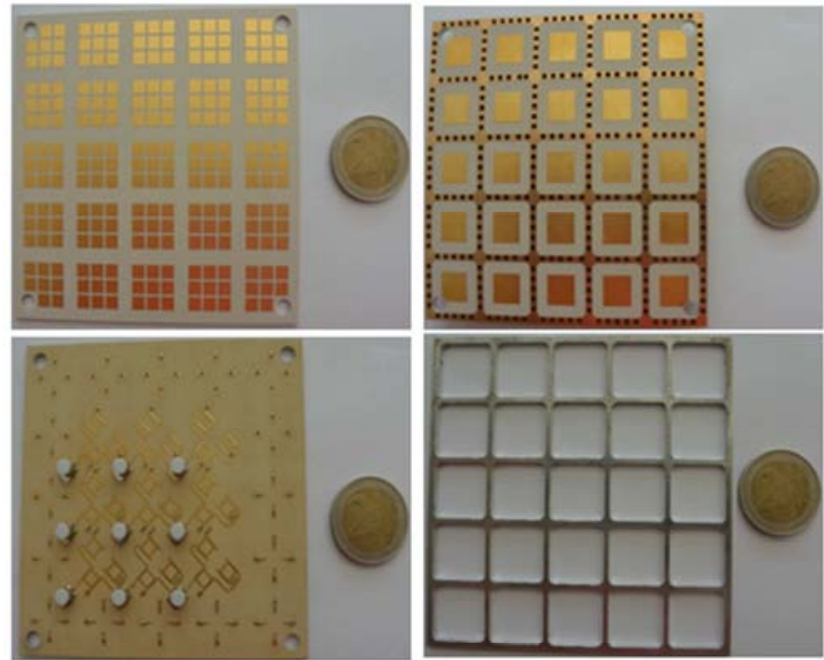

(a)

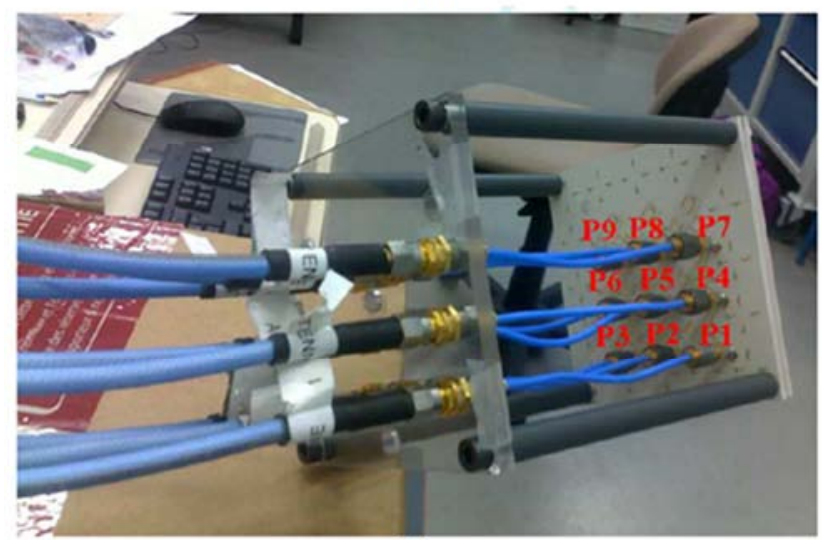

(b)

Figure 28. (a) FSS layer, patches layer, polarization circuits layer and metallic walls, (b) whole assembled design in measured by VNA. 


\section{Experimentations and Comparison}

\subsection{Power Divider Performances}

The measured $S_{j, 1}$ parameters show a good stability on a wide bandwidth $(1 \mathrm{GHz})$, meaning that the ratio of output magnitudes from the ports $2,3,4,5,7,8,9$ and 10 to the output magnitude at port 6 is maintained constant $(0.17)$. The same is for the phase shift $\left(180^{\circ}\right)$ required as shown in Figure 29. This insures the stability of the beam forming pattern required from this power divider over wide band.
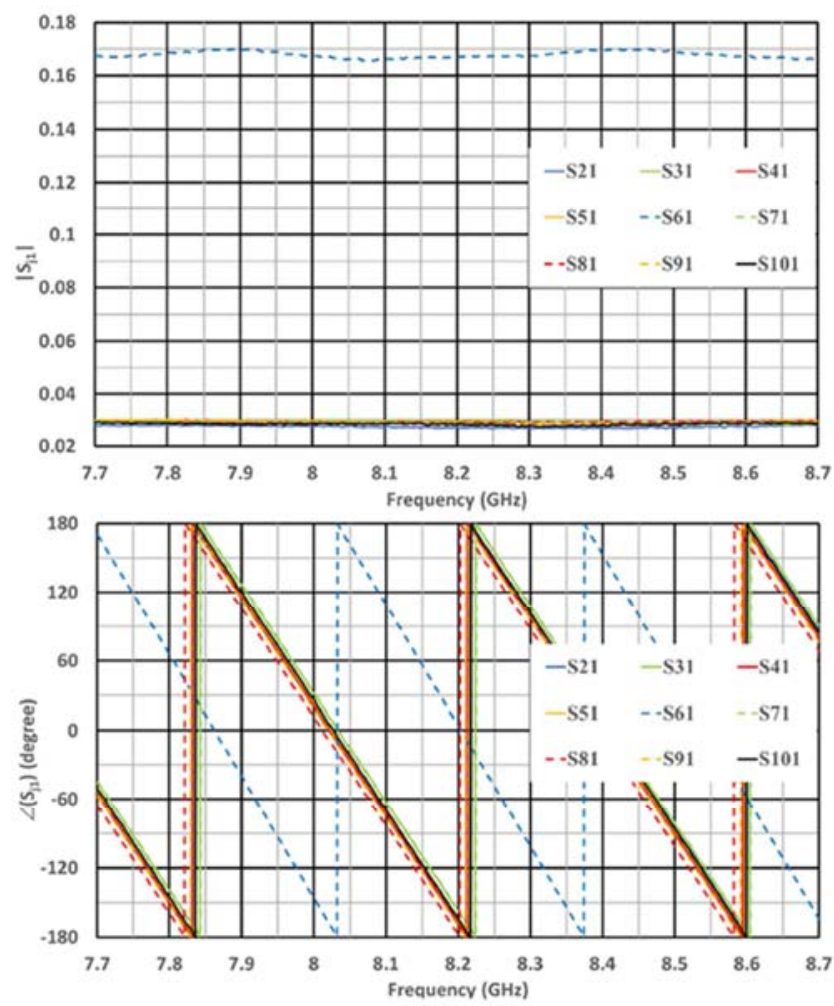

Figure 29. $S_{j, 1}$ Power divider outputs over $1 \mathrm{GHz}$ bandwidth, (top) magnitudes, (bottom) phases.

\subsection{Matrix Antenna Scattering Coefficients}

The measured and simulated $S_{\text {ii }}$ parameters are shown in Figure 30a and Figure 30b. In both cases, the curves show the $S_{\text {ii }}$ parameters lower than the expected "- $10 \mathrm{~dB}$ " value over $1 \mathrm{GHz}$ bandwidth illustrating the capacity of the ARMA solution to work on a wide frequency band. However, the $S_{i i}$ minima are shifted towards high frequencies in the experimental results.

A main property of the ARMA is that the couplings between the pixels are very small: usually smaller than the ones between elementary antennas in AESA [2]. This property is well illustrated in Figure 31 which shows the modulus of the coupling coefficients between the central port (P5 in Figure 28b) and the other ones for all the frequency range. The experimental results (Figure $2 \mathrm{~b}$ ) are lower than the theoretical ones (Figure 31a) because the losses are increasing; this phenomenon was expected.

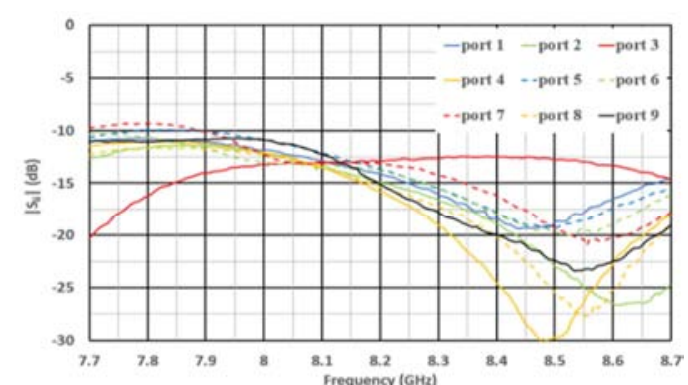

(a)

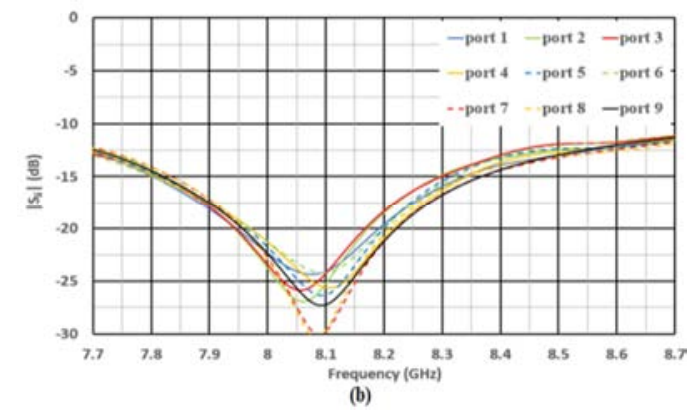

Figure 30. (a) Simulated return loss parameters, (b) measured return loss parameters, bandwidth of $1 \mathrm{GHz}$ below $-10 \mathrm{~dB}$.
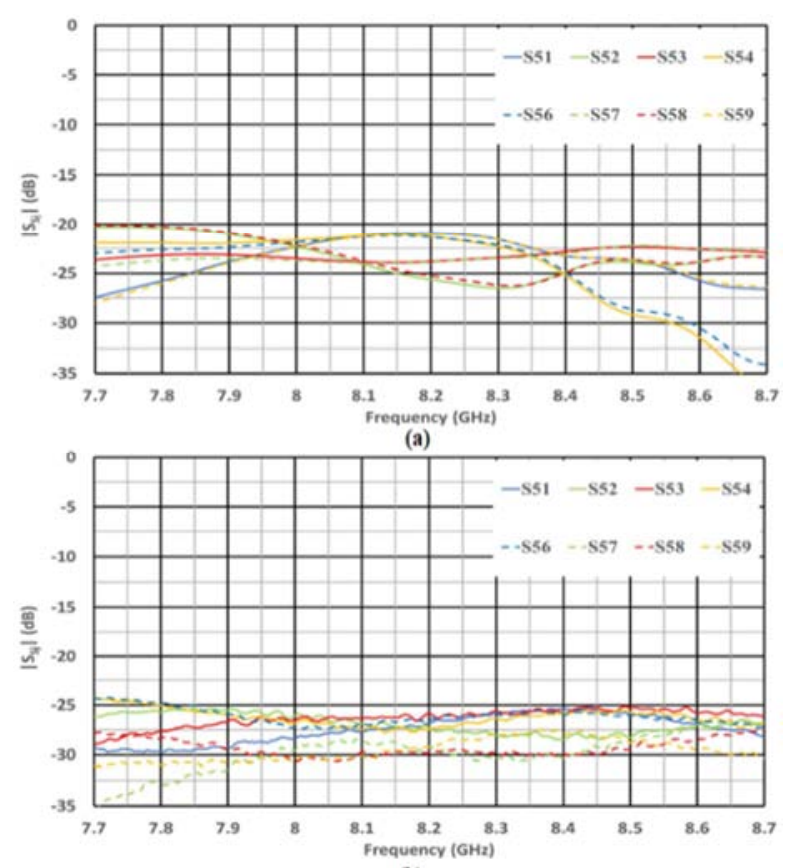

(b)

Figure 31. (a) Simulated return loss parameters, (b) measured return loss parameters, bandwidth of $1 \mathrm{GHz}$ below $-10 \mathrm{~dB}$.

\subsection{Gain Pattern}

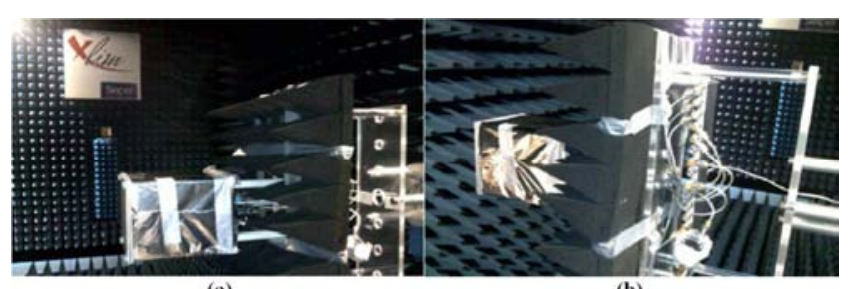

(a)

(b)

Figure 32. Antenna with thin aluminum cover in the anechoic chamber, (a) side view, (b) back view. 
The antenna matrix is connected to the CTOS power divider then the radiation patterns (directivity, IEEE gain and realized gains) are measured in our anechoic chamber at Limoges University (Figure 32). An aluminum thin sheet allows simulating the Nano-Sat platform. Absorbers were installed behind the antenna to minimize electromagnetic interferences of the motor. Therefore, the back radiation will be minimized resulting in a slight increase of the antenna directivity.

The 3D RHCP radiation pattern simulation-measurement comparison is presented at the central frequency $8.2 \mathrm{GHz}$ in

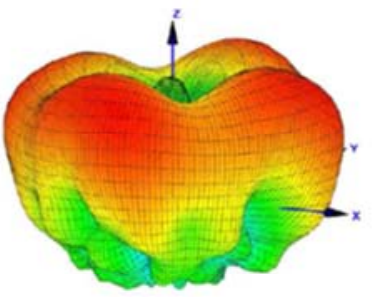

3D Simulated radiation pattern $6 \mathrm{dBi}$ at $8.2 \mathrm{GHz}$

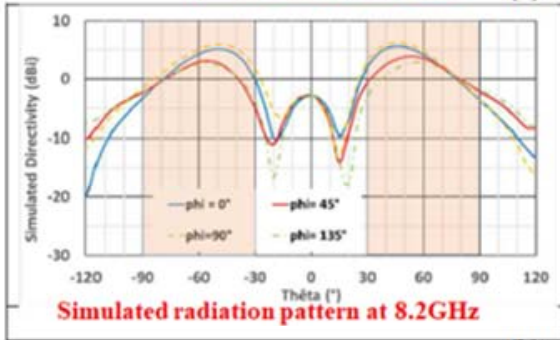

(b)

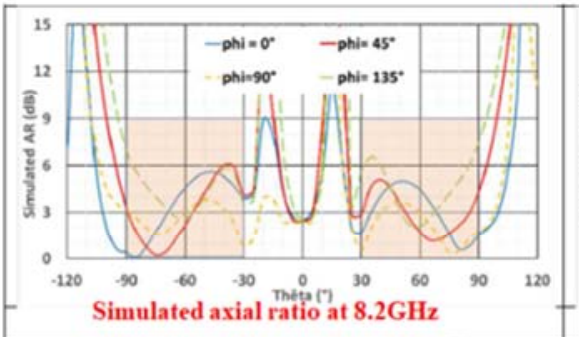

(c)

Figure 33. Simulation and measurement, (a) $3 D$ radiation pattern, ( $b$ and c) gain and axial ratio for $\varphi=0^{\circ}, \varphi=45^{\circ}, \varphi=90^{\circ}$ and $\varphi=135^{\circ}$.

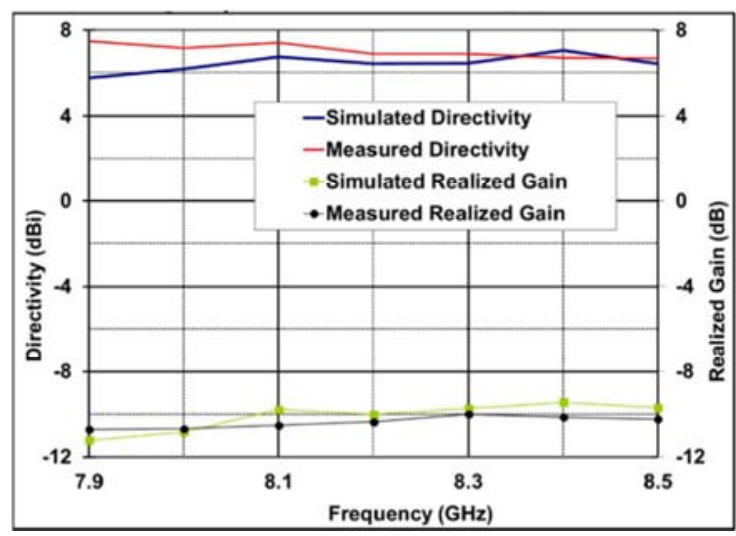

Figure 34. Theoretical and experimental directivity and maximum realized gain vs frequency.

Figure 34 shows the theoretical and experimental frequency evolution of directivity and associated maximum

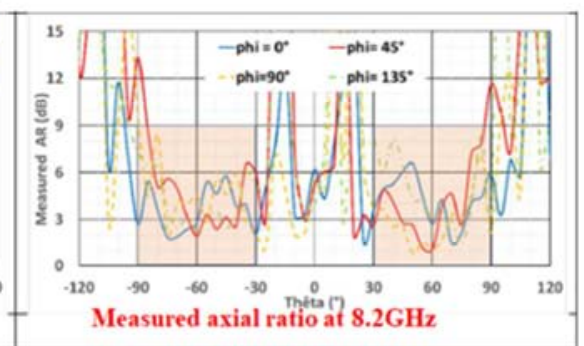

directivity in Figure 33a, the isoflux shape is illustrated on the $3 \mathrm{D}$ radiation pattern for simulation and experimental results. In both cases, the circular symmetry is perturbed by the antenna edges diffraction. Figure $33 \mathrm{~b}$ gives the RHCP radiation pattern in directivity at the same frequency for some $\varphi$ cut-planes $\left(0^{\circ}, 44^{\circ}, 90^{\circ}, 134^{\circ}\right)$ and Figure $33 \mathrm{c}$ shows the axial ratio function of the elevation angle. The results are not good around the axial direction but it is not a problem for spatial bi-mode applications [17] because the isoflux solution is used only for elevation angles higher than $\pm 30^{\circ}$.
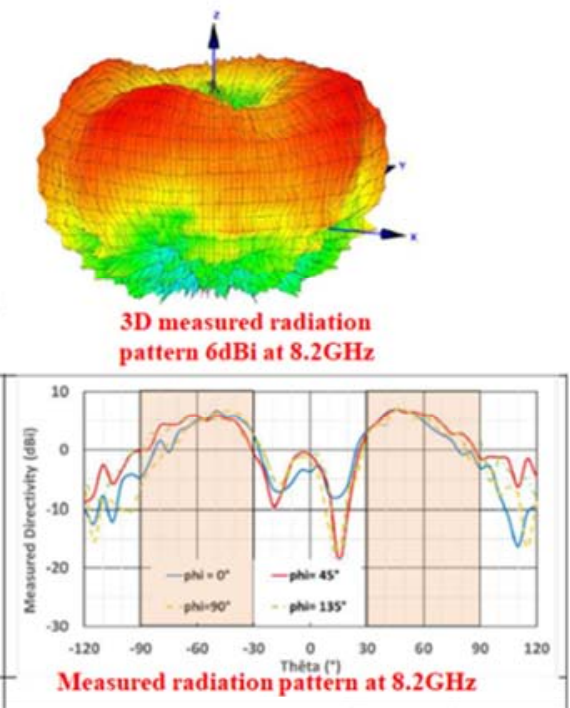

Measured radiation pattern at $8.2 \mathrm{GHz}$

realized gain. The theoretical and experimental results are in good agreement on the whole frequency band. The directivity is obviously higher than the realized gain (about $7 \mathrm{dBi}$ vs -10 $\mathrm{dBi})$ due to the losses of our experimental device introducing 14.4dB losses: $10 \log _{10}\left(0.17^{2}+8 \times 0.03^{2}\right)$. It remains $2.6 \mathrm{~dB}$ due to the losses in the antenna itself $(50 \Omega$ resistances, dielectric substrates, transmission lines, connectors...) and due to the experimentation process $( \pm 0.8 \mathrm{~dB})$.

\subsection{Nano-Satellite Body Effects}

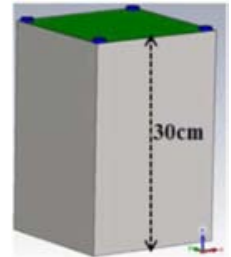

Figure 35. Matrix antenna with Cube-Sat in CST. 
The matrix antenna with the cube-Sat body is simulated in CST to see the performances like in real (Figure 35). Results show no effect for the cube-sat body on the gain and axial
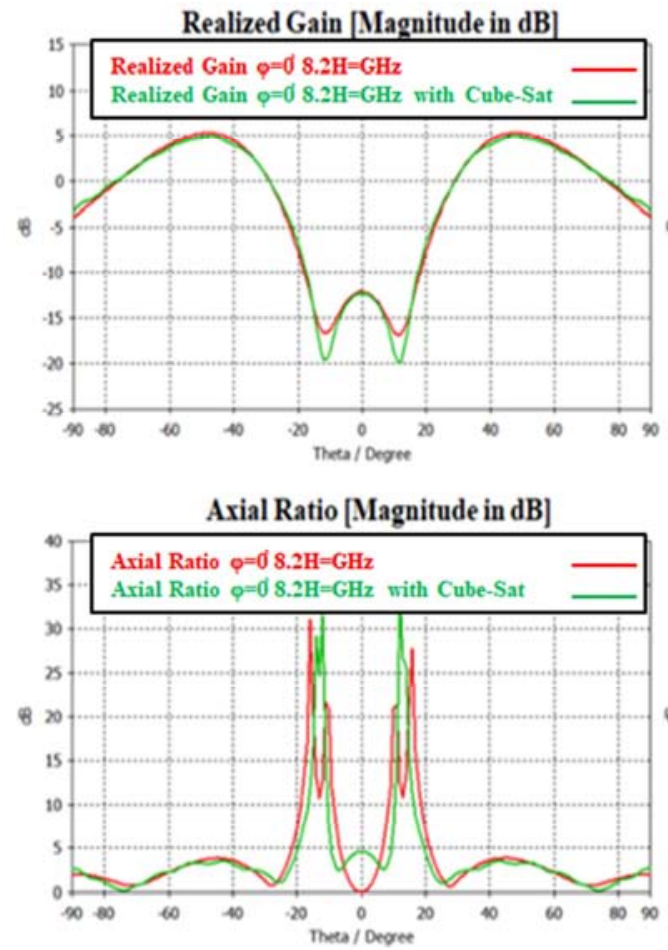

Figure 36. Cube-Sat effect (green with Cube-Sat and red without), (top) gain pattern, (bottom) axial ratio pattern.

\section{Conclusion and Perspectives}

In this review we addressed the problem of the bi-mode beam forming used in spatial telemetry links of the NanoSatellite technology. The designed antenna introduces the agility in beam reconfiguration using the new electromagnetic approach called ARMA. Using such a solution shows an enhancement in the formation of the beam with the maximum gain being at higher elevation angles (proved by the 1D matrix design). The isoflux thus attains the maxima at the angles less than $\pm 60^{\circ}$, but the gain at these elevation angles is no smaller than $1 \mathrm{~dB}$ compared to the maximum. Then the expected template is achieved, but the dissymmetry of the pattern in the different azimuth planes is axiomatic due to the square shaped matrix antenna. Also the axial ratio resulted gives the circular polarization constraint of the radiated beam even if at the high elevation angles.

Then the design of the antenna with the beam forming network solves the problems of the agility, small size, circular polarization and bandwidth. Also the bi-mode pattern is achieved where the switching angles are near $\pm 30^{\circ}$. The work was completed by fabricating the prototype and comparing the simulation with the measurements. The directive high gain beam was studied without measurements, but such a work just need to change the feeding network to obtain equally weights at the matrix inputs.

The challenges of the dissymmetry for now are resolved

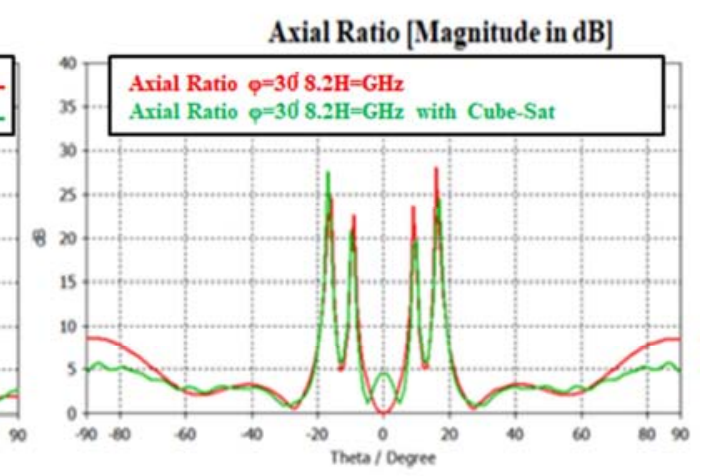

ratio patterns in the different azimuth planes. Figure 36 shows the comparison with and without Cube-Sat $(30 \mathrm{~cm}$ height) for the azimuth planes $\varphi=0^{\circ}$ and $30^{\circ}$.

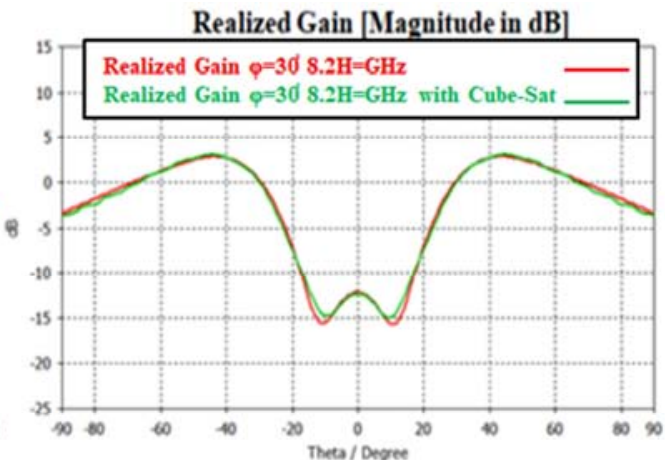

by optimizing the feeding law as show by the simulations. Future work prospect is to work on the physical structure of the matrix antenna itself, leading to an enhancement in the Omni-directionality of the wide beam and consequently fixing the maximum gain at wider elevation angles. Several works were done in the design of conformal matrix antenna using ARMA concept [17]. The challenge of the design of any shape pixel and then the matrix [17] validates the objectives needed to design a matrix antenna with different shapes to be fit the different applications physical constraints.

\section{Acknowledgements}

This work has been funded by the French Spatial Agency (CNES). Thanks for their efforts and fund that leads to complete this research and development.

\section{References}

[1] «Scan Performance and Configurability of Agile Radiating Matrix Antenna Prototype» H. ABOU TAAM, G. ZAKKA EL NASHEF, M. SALAH TOUBET, E. ARNAUD, B. JECKO, T. MONEDIERE and M. RAMMAL International Journal of Antennas and Propagation IJAP Vol. 2015 Article ID169303 8 pages. DOI: 10.1155:2015:169303.

[2] B. Jecko, E. Aranud, H. Abou Taam, A. Siblini The ARMA concept: Comparison of AESA and ARMA technologies for agile antenna design. Fermat Journal vol. 20, 2016. 
[3] Interests of a 1D EBG Matrix Compared to a Patch Array in Terms of Mutual Coupling and Grating Lobes. Hussein ABOU TAAM, Moustapha SALAH TOUBET, Thierry MONEDIERE, Bernard JECKO, Mohamed RAMMAL and Anthony BELLION: IEEE, 7th European Conference on Antennas and Propagation EuCAP, 8-12 April 2013, Sweden.

[4] Radiation control of an agile Matrix antenna by using specific algorithm. Hussein ABOU TAAM, Moustapha SALAH TOUBET, Thierry, Monedière, Bernard JECKO, Mohamed RAMMAL. IEEE, Mediterranean Microwave Symposium MMS, 2-5 September 2013, Lebanon.

[5] A Developed Excitation Law for Beam Forming And Steering Using A Novel Electromagnetic Band Gap Antenna. Hussein ABOU TAAM, Georges ZAKKA EL NASHEF, Eric ARNAUD, Thierry Monedière, Bernard JECKO, Mohamed RAMMAL. IEEE, 9th European Conference on Antennas and Propagation EuCAP, 12-17 April 2015, Portugal.

[6] TAAM, Hussein Abou, EL NASHEF, Georges Zakka, JECKO, Bernard, et al. Agile radiating matrix antenna system for radar applications. In: Radar Conference (Radar), 2014 International. IEEE, 2014. p. 1-4.

[7] «A New Agile Radiating System Called Electromagnetic Band Gap Matrix Antenna»: Hussein ABOU TAAM, Moustapha SALAH TOUBET, Thierry MONEDIERE, Bernard JECKO, Mohamed RAMMAL: International Journal of Antennas and Propagation, vol. 2014, Article ID 342518, 7 pages, 2014.

[8] Patent: JECKO, B.; HAJJ, M.; CHANTALAT, R.; SALAH TOUBET, «Antenne élémentaire et antenne réseau mono ou bidimensionnelle correspondante» PCT Patent: PCT/EP2012/076509; French Patent: FR 1162141.

[9] «New Circularly Polarized Matrix Antenna For Space Applictions» Ali SIBLINI, Bernard JECKO, Hussein ABOU TAAM, Mohamed RAMMAL WTS 2016 - London $18 / 04 / 2016$ au 20/04/2016.

[10] A. Siblini, B. Jecko and E. Arnaud, "Multimode reconfigurable nano-satellite antenna for PDTM application,"2017 11th European Conference on Antennas and Propagation (EUCAP), Paris, 2017, pp. 542-545. doi: 10.23919/EuCAP.2017.7928184.

[11] "Corners Truncated Microstrip Patch Antenna", Taha Imeci, Anil Saral Department of Electronics and Communication Engineering Halic University, Istanbul, TURKEY.

[12] R. Chantalat, L. Moustafa, M. Thevenot, T. Monédière and B. Jecko, "Low Profile EBG Resonator Antennas", Hindawi Publishing Corporation, International Journal of Antennas and Propagation, Volume 2009, Article ID 394801, 7 pages.

[13] Design of Hybrid Coupler. International Journal of Advanced Research in Electrical, Electronics and Instrumentation Engineering. T. Jayachitra, V. K Pandey and Anshuman Singh, Noida Institute of Engineering and Technology, Greater Noida, UP, India.

[14] L-band compact printed quadrifilar helix antenna with 'IsoFlux' radiating pattern for stratospheric balloons telemetry. Nelson Fonseca, Sami Hebib, Herv'e Aubert. HAL Id: hal00432239 .

[15] X-band compact dual circularly polarized isoflux antenna for Nano-satellite applications, Eric Arnaud, Cyrille Menudier, J Fouany, T Monediere, M Thevenot, International journal of microwave and wireless technologies, 2017, pp. 1-8, $<10.1017 / \mathrm{S} 1759078716001410>,<$ hal-01431624>.

[16] Experimental Validation of an Isoflux Earth Coverage with a Bi-mode ARMA Antenna on a Nano-satellite. Eric Arnaud, Ali Siblini, A. Bellion, B. Jecko. International Journal of Microwave and Wireless Technologies, Cambridge University Press/European Microwave Association 2019, MRF-RP-18418. R3.

[17] Agile Beam Radiating Surfaces, Bernard JECKO, Mohamad MAJED, Salma AIJA, Hassan CHREIM, Ali SIBLINI, Hussein ABOU TAAM, Joel ANDRIEU, Michele LALANDE, Edson MARTINOD. Forum for Electromagnetic Research Methods and Application Technologies Journal (FERMAT). Volume 30, Article 2, Nov.-Dec., 2018. 